\title{
Oxygenic photosynthesis: Critiquing the standing explanations and proposing explorative solutions based in murburn concept
}

\author{
Kelath Murali Manoj* \\ *Satyamjayatu: The Science \& Ethics Foundation \\ Kulappully, Shoranur-2 (PO), Palakkad District, Kerala State, India-679122. \\ Email: satyamjayatu@yahoo.com
}

\begin{abstract}
The available explanations for oxygenic photosynthesis for the light reaction of photolysis and photophosphorylation (Pl-Pp) are critically analyzed. Based on the structure cum distribution of protagonist molecules and the new mechanistic explanations in redox biochemistry, the interactive dynamics of key reactants are re-assessed for viability. The Zscheme (for electron transfer) and Kok-Joliot cycle (for water-lysis) are found to be physiologically non-viable. Further, mechanistic explorations based on murburn concept are advocated for Pl-Pp.
\end{abstract}

Keywords: murburn concept, photophosphorylation, photolysis, photosynthesis, light reaction,

1. Introduction

\section{Contents}

1.1 Anecdotes highlighting the complexity of photosynthesis research

1.2 Recent developments and the context of the current write-up

2. A succinct overview of the prevailing awareness on photolysis-photophosphorylation (Pl-Pp)

2.1 Compositional and structural aspects

2.1.1 PS II and cytochrome $b f /$ plastocyanin: Photo-hydrolytic machinery

2.1.2 PS I and ferredoxin/NADP reductase: Capture of redox equivalents

2.1.3 ATP-synthesizing complex: Phosphate coupling to ADP

2.2 Prevailing mechanistic assumptions and explanations

2.2.1 Interaction of light with plant pigments

2.2.2 Electron transfer chain and oxygenesis

2.2.3 Inhibition of Pl-Pp

2.3 Functional energetics

3. Critiquing the prevailing explanations for Pl-Pp

3.1 Little evidence or scope for proton pumps and proton-based ATP-synthesis

3.2 Structure-function correlations and evolutionary perspectives

3.3 Analysis of the Z scheme \& Kok-Joliot cycle

3.4 Inquiry into the energy-transfer mechanism between pigments \& overall energetics

3.5 The Emerson effect experiment

4. Redirecting pointers from relevant/analogous systems

4.1 Comparison of oxidative phosphorylation (OxPhos) and Pl-Pp

4.2 Cyanobacterial OxPhos \& Pl-Pp system for metabolic synchronization

4.3 Uncoupling proteins' / additive molecules' roles in mXM-OxPhos-Pl-Pp systems

4.4 Bacterial rhodopsin and the "chemical trapping" of light energy

5. Conclusions 


\section{Introduction}

Photosynthesis is a complex cascade of reactions salient of green plant cells, which leads to the light-assisted splitting of water and fixation of carbon dioxide, forming simple organic sugars. Scientists believe that since the past two and a half billion years, photosynthetic reactions serve as the ultimate earthly molecular source and the mainstay of the "fuel" used by most life forms in cellular respiration. On the other hand, cellular respiration, also called (mitochondrial) oxidative phosphorylation or $(\mathrm{m}) \mathrm{OxPhos}$, is the redox metabolic scheme that consumes reduced substrates using oxygen, to synthesize energy equivalents of ATP. At the global level, mOxPhos serves as the fundamental "engine" that drives cellular activities, by "burning" the fuel that photosynthesis generates. Seen from the thermodynamics perspective- while cellular respiration is an overall downhill process, the photosynthetic reaction is an overall uphill process. On our planet, the latter "potential building" chemical process is enabled primarily by photolysis (Pl, lightsponsored splitting of water, which serves as the source of electrons for generating the reduced nicotinamide nucleotide product, NADPH) and photophosphorylation ( $\mathrm{Pp}$, light-enabled addition of phosphate to ADP, thereby generating the chemical energy currency of ATP) reactions. These reactions obligatorily require light energy and occur within sub-cellular organelles called chloroplasts (a type of plastid) of plant cells. Therefore, chloroplasts harvest the energy of sunlight to synthesize redox equivalents (NADPH) and chemical energy reserves (ATP) that are subsequently utilized for the actual carbon dioxide fixation reaction (called the Calvin-Benson cycle, which does not need light).

\subsection{Anecdotes highlighting the complexity of photosynthesis research}

We have come a long way in understanding several intriguing aspects of photosynthesis, thanks to the pioneering works of several researchers, who worked tirelessly on the daunting problem over decades. Herein, it is worth pointing out two specific historical controversies that deal with the fundamental theoretical and experimental premise/crux of photosynthesis.

1. Counting photons required for the formation of an oxygen molecule: Quite like the tussle of views between Peter Mitchell and Bill Slater regarding OxPhos, two stalwarts of Pl-Pp research, Otto Warburg and Robert Emerson, sparred on some fundamental mechanistic aspects. Warburg was a renowned biochemist and a Nobel laureate, who was nominated for the much-coveted prize a record 47 times within his lifetime. In the pertinent field, he is known for the Warburg effect, which concerns the lowered photosynthetic yield at high oxygen concentrations. On the other hand, Emerson (Warburg's doctoral student) had commissioned some original/pioneering quantitative studies in the field when he found that 2500 chlorophyll molecules form a photosynthetic unit. (Restated- maximum efficiency of oxygen formation resulted when one of $\sim 2500$ chlorophyll molecules absorbed a quantum of light.) He also championed the Emerson effect (Emerson et al., 1957), which proposed that two distinct photosystems existed in chloroplasts and these two distinct systems function in a synchronized physiological pathway for the acyclic photoylsis-photophosphorylation (Pl-Pp). [This was captured as the "Z-scheme" of ETC first by Hill and Bendall in 1960. Several other researchers also contributed in significant ways towards the establishment and popularization of the "Zscheme" (Govindjee, 2017). However, Emerson can be rightfully deemed as the pivotal 
axis for the development of the concept of "coupled photosystems within physiological Pl-Pp ETC'.] The bone of contention (which did not end with Emerson's untimely death in 1959; and which Warburg carried on until his own demise in 1970) between WarburgEmerson duo was on a very fundamental quantitative issue. While Warburg believed that $\leq 4$ photons were enough to run the photolytic 4-electron acyclic scheme of oxygen evolution, Emerson believed that $\geq 8$ photons were required for the same. Emerson's 8photon perspective prevails today through the "linear electron transport of the Z-scheme". [Warburg could perhaps have indirectly indicated reconciliation with Emerson's proposal in his last paper (Govindjee, 1999).]

2. The mechanism of relay of photons orland their energy to the reaction center: The "energy transfer" from the supposed light harvesting complexes and multitude of pigments scattered within the thylakoid membrane to the reaction center deep-seated within the photosystems has been a center of intense theoretical and experimental investigations. The pioneer James Franck (of Franck-Condon principle fame) had argued in late 1930s that "exciton transfers" would not be possible within living photosynthetic systems. It was only several decades later that Franck's "gauntlet" was picked up by the researchers of a later generation, countering him, and advocating that exciton transfer was both a theoretically and experimentally "viable" mode for photosynthetic energy relay (Bay \& Pearlstein, 1963; Philipson \& Sauer, 1972). As this essay is being written, experts in the field indulge three distinct modalities of energy transfers (Panitchayangkoon et al., 2011)-

(i) excitonic hopping [classical resonance energy transfer; wherein energy is transferred from one electronic state of a chromophore to the next. Herein, the "hops" indicate a rather particle type attribute that the energy has to assume to travel from its site of absorption along a random or predetermined route, to the destination of Chla-RC (reaction center)],

(ii) wave-like propagation [coherence transfer; wherein a quantum mechanical phase relationship exists/persists between the participants. Rather than behaving like a particle that travels in a given route, the energy packet absorbed behaves like "a quantummechanic wave-spread", thereby sampling all possible routes simultaneously/instantaneously.], and

(iii) quantum transport [which involves a coupling of both population and coherence dynamics, leading to a type of oscillatory paradigm].

The details of these two instances are quoted to impress upon the reader(s) that the field is very complex and highly challenging, both theoretically and experimentally. It is clear that there has been a dearth for consensus regarding mechanistic underpinnings and even seasoned researchers working in the area have been given to confusions and misunderstandings. The Editorial of the leading journal Science (Volume 340, Issue 6139, 21 June 2013, page 1373), while introducing two manuscripts within the particular issue, opines in the contents section- "It is unclear how energy absorbed by pigments in antenna proteins is transferred to the central site of chemical catalysis during photosynthesis." Caffarri et al. (2014), in a recent review, say- "Although many aspects of the photosynthetic process are nowadays substantially elucidated, several details, specific regulations, and even structural details about photosynthesis in plants are still little known." In the abstract of a key structure elucidation paper, Wei et al. (2016), write- "The 
pathways along which excitation energy is transferred between them (reaction center and LHC), and their assembly mechanisms remain to be deciphered......." These statements strongly consolidate the fact that photosynthetic mechanisms are still mysterious, in many ways.

\subsection{Recent developments and the context of the current write-up}

Over the last decade, through a reductionist approach, select pursuits have unraveled new mechanistic insights on the "radical" way in which certain redox enzymes and their metabolic pathways could orchestrate and function, particularly in the vicinity of phospholipid membranes (Manoj \& Hager, 2006; Manoj \& Hager, 2008; Manoj, Baburaj et al., 2010; Manoj, Gade et al., 2010; Andrew et al., 2011; Gade et al., 2012; Gideon et al., 2012; Parashar \& Manoj, 2012; Parashar, Gade et al., 2014, Parashar, Venkatachalam et al., 2014; Manoj, Gade et al., 2016; Manoj, Parashar, Venkatachalam et al., 2016; Manoj, Parashar, Gade et al., 2016; Manoj, Venkatachalam et al., 2016; Venkatachalam et al., 2016; Manoj, 2017; Parashar et al., 2018; Manoj, 2018; Manoj, Parashar et al., 2018; Manoj, Gideon et al., 2018). These works have overhauled key perspectives in the reaction mechanism of diverse ecologically relevant (halo)peroxidases, mixed function heme/flavin containing cytochromes/reductases of liver microsomal xenobiotic metabolism (or mXM), and the cascade of heme/flavin/Fe-S/Cu proteins of mitochondrial/prokaryotic oxidative phosphorylation (m/p OxPhos). Such developments have also explained intriguing physiologies like biothermogenesis and hormetic/idiosyncratic doseresponses.

Given the upheaval in the field of redox metabolism and the fact that mechanistic chemistry is considered an enigmatic "black box" should inspire us to challenge the pertinent "accepted/textbook" ideas, and probe all assumptions/interpretations with meticulous zeal. Also, the structures of various components of the photophos ETC (electron transport chain) and the light harvesting complex (LHC) were recently revealed (Benson et al., 2015; Wei et al., 2016; Young et al., 2016; Mazor et al., 2017). Therefore, the mandate to critically inspect whether these new information fit well with age-old perspectives is fully warranted.

The current manuscript deals with the mechanistic purview of the first part of photosynthesis, namely: the light reaction involving photolysis-photophosphorylation (hereon called Pl-Pp). Specifically, I shall focus on the quantitative aspects of structure-function correlation and "space-time-yield" of Pl-Pp process. Further, the overall mechanism/phenomenology by which chloroplasts lyse water to evolve oxygen, and by which ATP cum NADPH synthesis occurs therein are also discussed. I shall first present a succinct overview of the current Pl-Pp awareness [as available from leading reviews and textbooks of Lehninger, Stryer and Voet (Lehninger et al., 2004; Berg et al., 2002; Voet \& Voet, 2011)], and then establish the clear rational premise for challenging the prevailing notions.

\section{A succinct overview of the prevailing awareness on PI-Pp}

Before proceeding to the critical dissection, a summative perspective of the Pl-Pp system is first provided so that we can have a solid foundation on the known facts that we need to build on. The contents gathered herein are predominantly from the three popular textbooks of Lehninger, Stryer and Voet. Further, Govindjee's book "Bioenergetics of Photosynthesis" (Govindjee, 1975) 
would also clearly give the reader good chronological and current perspectives. Many of the fundamental works (extracted and re-presented from the sources above) leading to the current awareness are not cited with references here, and this intentional "faux pas" is necessary to save space. [[If the reader seeks a more elaborate background on the prevailing biophysical concepts and awareness on the subject, I would strongly recommend the contents of Antony Crofts' and Govindjee's websites at UIUC and select papers cited therein-

(http://www.life.illinois.edu/crofts/bioph354/)

(http://www.life.illinois.edu/govindjee/g/TeachingMaterial.html).

The details on ETC and oxygenesis presented herein are majorly drawn from their writings/edited write-ups. For a different perspective, one could also avail a brilliant "self-study" of photosynthesis by Donald E. Fulton, a retired electrical engineer, who ventured to write on the subject while recovering from a debilitating bout of cancer. His write-up can be found athttp://www.twinkletoesengineering.info/photosynthesis.htm]]

\subsection{Structure and composition of chloroplasts}

Photosynthesis occurs in chloroplasts, which have a linear dimension of 1 to 10 microns; 5 microns being the average 'size' of these lipid-membrane ensconced organelles. A plant cell could have anywhere from one to thousand chloroplasts and each of these plastids has a continuous aqueous core called stroma. Embedded/dispersed in the stroma are found highly stacked self-folded vesicular structures (ranging in 20 to 100 per chloroplast) called grana (with linear dimensions of 500 to $800 \mathrm{~nm}$ ), which are inter-connected through slender membranous lamellae. Each granum may comprise of several thylakoids, which are flattened vesicles with very minute amounts of "aqueous lumen". Thylakoid membranes could be 4 to $10 \mathrm{~nm}$ thick and the lumen could be 10 to $50 \mathrm{~nm}$ across at the narrow sides. The thylakoids membranes are low on lipids and high in proteins, quite like the inner membrane of mitochondria. However, in the thylakoid membranes, up to $80 \%$ of the lipids belong to the unsaturated galactolipids class (quite unlike phospholipids like cardiolipin that are predominantly found in mitochondria).

Thylakoids can be deemed as a fundamental photosynthetic unit because they host almost all of the various protein/pigment machineries involved in photo-excitation, electron transport and ATP/NADPH synthesis - as respectively exemplified by Light Harvesting Complexes (LHCs), Photosystems, Complex V, etc. These components may also have preferential distributions of various elements within (a general schematic is shown in Figure 1). For example- PS II may be present in greater abundance within the stacked region of grana, versus the lamellar connective. Further, the distribution ratios of various elements may also vary depending on the cell's location on the leaf, growth phase, etc. For example- the cells on the leaf's under-side may have a different distribution of photosystems and LHCs compared to the upper-side. [In the context of critical discussions within this write-up, the word photosystem shall henceforth refer/connote primarily to a protein-cofactor multi-molecular assembly which efficiently carries out a oneelectron charge separation, giving rise to a two-electron stable product. Therefore, this definition may or may not include some non-essential Chlorophyll Binding Proteins (CBPs) or LHCs.]

Figure 1: A cross-section through a granum to show the approximate schematic arrangement of Pl-Pp machinery. (Adapted unabashedly from Caffari et al, 2014) The diagram gives a rough depiction of the location, orientation, and relative distribution densities of the participants. The membranes of thylakoid are $\sim 4 \mathrm{~nm}$ thick and PSII+LHCII complex is $\sim 17 \mathrm{~nm}$ across. Plastocyanin (not shown herein) is 
supposedly located within the continuous blue line (lumen of thylakoids). The NADP reducing components (not shown herein) of Ferredoxin is found soluble within the stroma and NADP reductase is believed to be located in the stroma as well as the thylakoid membrane. The stromal (thick blue phase) and thylakoidal (thin blue lines) aqueous phases are disconnected. The lumen thylakoid lipid bilayer membranes have copious amounts of quinones (not shown).

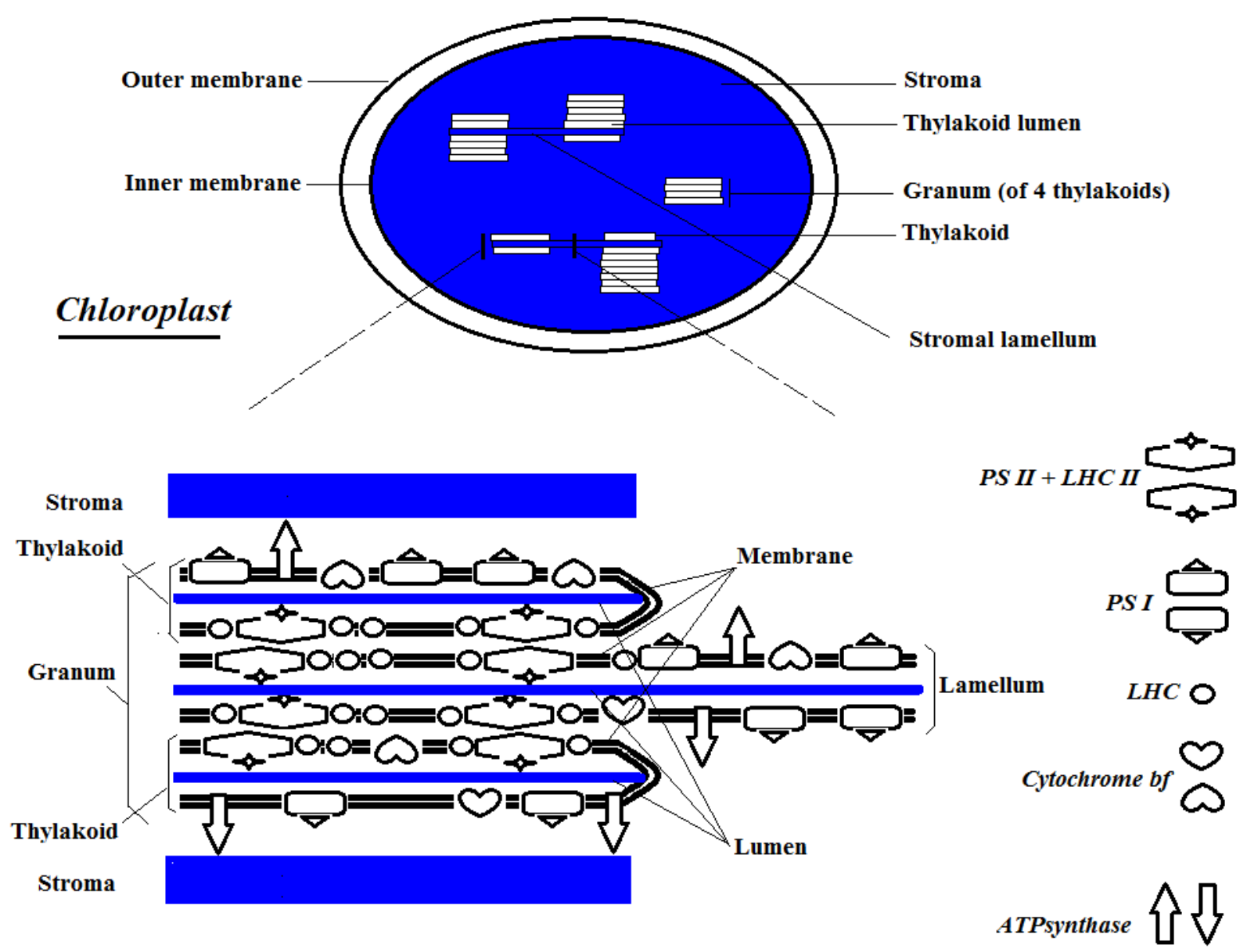

A chloroplast may have hundreds thylakoids, and each thylakoid comprises of hundreds or thousands of photosystems and other accompanying proteins/complexes that are required for PlPp pathway. Each photosystem may be multimeric with several tens of protein subunits, along with its dedicated CBPs and LHCs. In toto, the PhotoSystem Core - Chlorophyll Binding Proteins - Light Harvesting Complexes (PSC-CBP-LHC) incorporates hundreds of pigments and cofactors belonging to various classes. Therefore, a chloroplast would have thousands to millions of copies of various pigment molecules like chlorophylls, carotenoids, xanthophylls (lutein), etc. All pigments are predominantly hydrophobic and characterized by an extended linear or cyclic pi-electron cloud, with alternating single and double bonds. There appears to be little "tangible logic" in the spatial arrangement and geometrical placement of various light harvesting pigments around the reaction centers of photosystems I and II. Both Photosystems I \& II are associated with $\sim 200$ chlorophyll and $\sim 50$ carotenoid molecules. An LHC II typically contains 7 Chla, 5 $\mathrm{Chl} b$ and 2 lutein. Such an LHC may function as a trimer, closely associated with either PS I or PS II. Both PS I and PS II may have several LHC I and LHC II alongside, present in different spatial arrangements, in conjunction with the CBPs. (Herein, it is not deemed necessary to get 
into the details of the names and positions of the different proteins.) Generally, most plants have a Chla:Chl $b$ ratio of 2. The PS I / PS II chlorophyll ratios have been reported to vary from 0.54 to 1.4; usually averaging at about 1 . (For more details and specifics on distribution of the pigments in various systems, please refer Caffarri et al., 2014.)

The structural components of the Pl-Pp system for plants can be studied under three distinct heads (and they are presented in the order of the perceived sequence of reaction)-

\subsubsection{Photosystem II \& cytochrome bf-plastocyanin: The photo-hydrolytic machinery}

This part of the Pl-Pp machinery is purportedly analogous to the photosynthetic ETC system present in purple bacteria $\left(\mathrm{P} 870+\mathrm{Cyt} b c_{1}\right.$ complex + Cyt $\left.c_{2}\right)$, but without the facet of photolysis of water. In plants, the proteins within this system supposedly use light energy to generate proton gradients and use water as a source of electrons.

Within the PS II multi-protein complex system, up to $50 \%$ of the chlorophylls belong to Chl $b$ type. Chlorophylls $a$ and $b$ differ in their absorption spectrum because of the presence of a carbonyl substitution in the latter. The carotenoids primarily belong to the carotenol or xanthophyll type. PSII is a heterodimer with approximately 3 dozen Chl $a$, a dozen $\beta$-carotene, two pheophytin, two plastoquinone, two heme, two non-heme Fe, etc., and a pivotal $\mathrm{Mn}_{4} \mathrm{CaO}_{5^{-}}$ $\mathrm{Cl}_{2}$ cluster. The photo-reaction center of this system is Chla dimer (absorbing light at the wavelength of $680 \mathrm{~nm}$, the reason why this reaction center is also called P680), although only one monomer is supposed to be catalytically active. The Mn-cluster is considered as the crucial element that holds together two water molecules, forging covalent bond formation between oxygen atoms. Approximately, 10 plastoquinone (PQ) molecules exist per RC (reaction center).

Cytochrome $b f$ complex is a dimer, each monomer being made of several subunits and prosthetic groups $(d+$ pi conjugated electron systems like hemes, $d$ electronic systems like Fe-S clusters; besides pi electronics like chl $a$ and $\beta$-carotene). This complex protein is the purported center of the "Q cycle" and interactive hub of PS II and PS I activities.

Plastocyanin is a soluble small protein with copper as cofactor. It is believed to be the crucial electron relay agent between PS II (via Cytochrome $b f$ ) and PSI. The surface topography/electrostatics of plastocyanins vary for plants, algae and cyanobacteria but the copper ligation microenvironment is more or less conserved.

\subsubsection{Photosystem I \& Fd-NADPreductase: Capture of redox equivalents}

These proteins are functionally analogous to the photosynthetic ETC system present in greensulfur bacteria (P840 + Ferredoxin-NADPreductase). In plants, proteins within this system are supposed to use light energy to cycle the electrons availed from PS II, in order to reduce NADP ${ }^{+}$.

Within the PS I system, there are only a few Chl $b$ and the chlorophylls are almost fully of Chla type. The carotenoids are majorly of $\beta$-carotene class. The reaction center is again made of Chla dimers, which absorb light at $700 \mathrm{~nm}$. A modified chlorophyll $\mathrm{A}_{0}$ acts as the first electron acceptor, which is subsequently passed on to phylloquinone and $\mathrm{Fe}-\mathrm{S}$ reaction centers. 
Ferredoxin is a small soluble protein with an active $2 \mathrm{Fe}-2 \mathrm{~S}$ center and this small protein is known for its promiscuity in serving as electron transfer agent in a wide variety of reaction environments. Finally, NADP reductase is a flavoenzyme with FAD as cofactor. This enzyme takes two electrons (one each from two reduced ferredoxin molecules) to pass on to NADP ${ }^{+}$, giving NADPH as the final reduced product in Pl-Pp cascade.

\subsubsection{ATP-synthesizing complex}

The ATPsynthase of chloroplasts has the $\mathrm{F}_{1}$ bulbous part (with affinity for ATP) sticking out into the stroma, with its $\mathrm{F}_{\mathrm{o}}$ domain (a proton conducting channel) tethered on to the thylakoid membrane. A controlled movement of 3-4 protons through the $\mathrm{F}_{\mathrm{o}}$ domain (from the lumen, into the stromal side) supposedly leads to a single ATP molecule's synthesis.

\subsection{Prevailing mechanistic assumptions and explanations}

\subsubsection{Interaction of light with chloroplast pigments}

Chla absorbs light at $430 \mathrm{~nm}$ and $662 \mathrm{~nm}$ whereas Chl $b$ absorbs light at $453 \mathrm{~nm}$ and $642 \mathrm{~nm}$. These abundant pigments absorb in the blue and red wavelength ranges, and therefore appear green. Carotenoids absorb between $460 \mathrm{~nm}$ and $550 \mathrm{~nm}$ and appear orange-red. As per the prevailing mechanistic perspective, a pigment molecule within the photosystem or LHC absorbs a photon (corresponding to a quantum of energy) and the energy from this photon could be theoretically relayed to the reaction center Chl $a$ via two fundamental modalities-

(i) A radiative energy transfer by the emission of a photon of higher wavelength (by the phenomenon of fluorescence), which another chromophore could absorb and utilize.

and/or

(ii) A non-radiative and sort of conductive/convective energy transfer (a kind of dipoledipole quantized migration) of an "exciton" (one modality of which is the wellestablished Forster transfer).

The current mechanistic perspectives vouch that option (i) is rare but option (ii) is preponderant. That is- a series of exciton transfers or migrations (functionally equivalent to the transfer of energy quanta) occurs before an actual electron transfer step takes place at the reaction center. Across the bacterial and plant systems, the number of elements in a photosynthetic unit (the number of pigments like chlorophyll involved in the absorption of definite light quanta, leading to the evolution of a single oxygen molecule or reduction of $\mathrm{CO}_{2}$ molecule) does not conform and are found to vary from a few tens to a few thousands (50-5000; Emerson found the average number to be 2500). The exciton transfer is supposed to occur from LHC II to LHC I / CBP to reaction center Chl $a$, via multiple pigments (chlorophyll $b \rightarrow$ lutein $\rightarrow$ zeaxanthin $\rightarrow \beta$-carotene $\rightarrow$ lycopene $\rightarrow$ chlorophyll $a$ ), at a fantastic $>90 \%$ efficiency, in a timescale of $10^{-10}$ s. The lightinduced electron transfer is supposed to occur only at the reaction centers of PS I and PS II. The current Z-scheme of "8 photon - 2 water molecules - 1 molecule of oxygen (4 protons)" 
translates to an approximate value of $\sim 2400$ chlorophylls per oxygen molecule evolved, $\sim 600$ molecules of chlorophylls per electron/proton transferred and $\sim 300$ chlorophyll molecules per photon absorbed.

\subsubsection{Electron Transport Chain (ETC) and the evolution of oxygen}

ETC: As discussed in the introduction, purportedly, there is an elaborate scheme of electron transport chain within the chloroplasts, which is depicted in Figures 2 and 3. The electron must first travel from the WSC located in the thylakoid lumen side to the membrane-embedded RC of PS II. From there, it is supposedly relayed via a series of fixed and mobile agents (located distal to the WSC side) to reach the heme x side of Cyt $b f$. From there, it is supposedly relayed through several centers to heme $f$ (present by the lumen side), where from plastocyanin collects the electron, to be relayed to the lumen side of PS I. Now, the electron goes up again towards the stromal side, to be picked up by ferredoxin-NADP reductase system. PS II and Cyt $b f$ complexes are also supposed to act as proton pumps, ultimately serving the rotary ATP-synthase.

There are 3 legs (Phases) of downhill electron transfer steps. The Phase I (water to ChlP680 ${ }^{+}$) ET is currently supposed to involve the intermediacy of at least two relay centers; Water $\rightarrow$ MnComplex $\rightarrow$ Tyr $\rightarrow \mathrm{P} 680^{+}$. Phase II $\left(\right.$ChlP680* to ChlP700 $\left.{ }^{+}\right)$is currently supposed to involve several relay centers; P680* $\rightarrow$ Pheophytin $\rightarrow$ Bound \& Free plastoquinone molecules $\rightarrow$ FeS and heme centers of Cyt bf complex $\rightarrow$ Plastocyanin $\rightarrow \mathrm{P} 700^{+}$. Finally, Phase III (Chlp700* to $\mathrm{NADP}^{+}$) is also supposed to involve several relay centers; P700* $\rightarrow$ ChlA $\rightarrow$ Phylloquinone $\rightarrow$ Three Fe-S clusters $\rightarrow$ Ferredoxin $\rightarrow$ NADP reductase $\rightarrow$ NADP $^{+}$. It must be born in mind that all phases start with an electron-paired non-radical species and go through a series of oneelectron transfers. In Phases II and III, stable two-electron species are supposed to be formed in the quinone intermediates. Also, there are two obligatory photon-induced uphill excitation steps (Zap I = ChlP680 to ChlP680* and Zap II = ChlP700 to ChlP700*) that make the overall "roller coaster" ride of the electrons possible. In the scheme, water molecules within the thylakoids serve as the source of electrons and the electron sink is supposed to be NADP ${ }^{+}$present within stroma. Since photosynthetic yields derived from the working of PSI or PSII independently were lower when compared to the combined system, this elaborate Z-scheme was deemed to be the "physiological ETC".

Figure 2: A visualization of the "actual path" and sequential transfer for the Pl-Pp ETC. The diagram is only a rough schematic approximation of the most favorable considerations for dimensions. The continuous arrows are ETs within a protein complex or within a donor-acceptor supercomplex. The dashed arrows show "migration of a diffusible electron carrier". A half arrow shows the transfer of an electron whereas a full arrow is the transfer of electron pairs. As seen, within the two photosystems, there are both one and two electron transfer schemes. After starting off from the lumen (in water), the electrons take refuge in the membrane, to return to the lumen (carried on PC), ultimately traversing the membrane into the stromal side. $(F d=$ ferredoxin, $N R=N A D P$ reductase $)$ Trans-membrane proton potential $(T M P)$ across the thylakoid membrane is depicted on the left with-ve and +ve signs. 


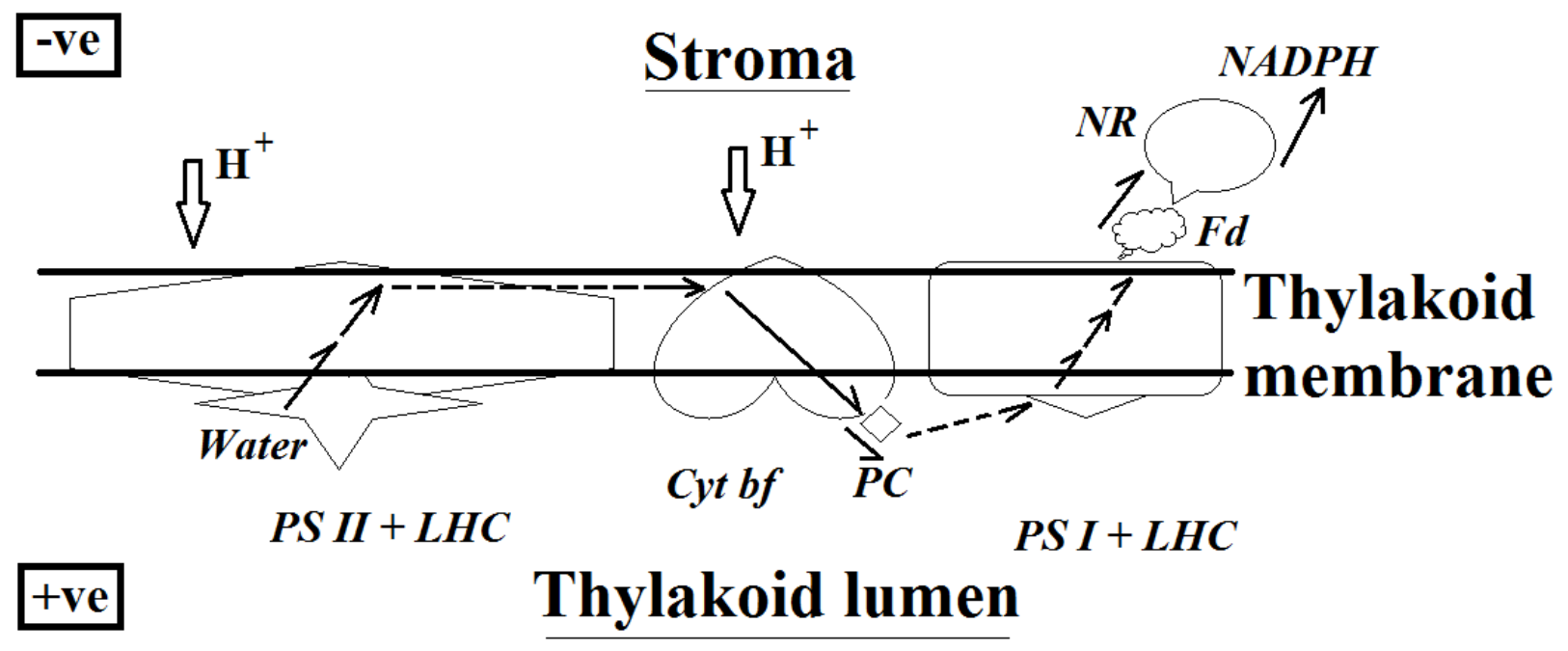

Figure 3: A depiction of the prevailing acyclic electron transfer scheme of Pl-Pp. The redox potentials of listed components are merely a rough depiction, and the boxed entries are not included within the purview of the $Y$ axis. The number of breaks within a phase arrow indicates the approximate number of currently presumed transient relay centers or intermediates. The formation of stable intermediates is indicated with a full arrow. The formation of intermediary stable two-electron products of hydrogenated quinones (within Phases II and III) are not depicted herein. The cyclic intermediates of the RC have been expanded to show the details with respect to electron reception and donation.

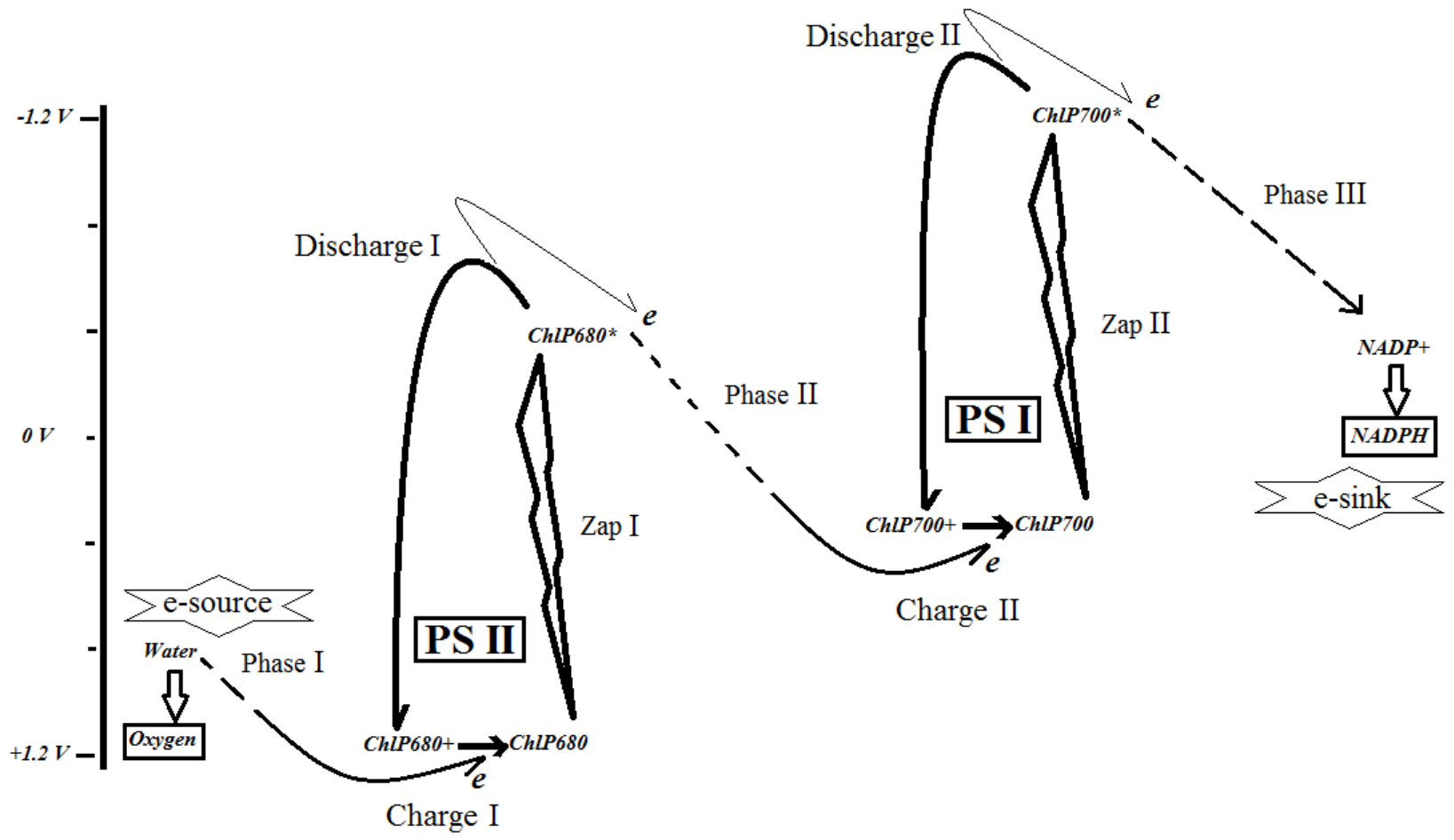


Oxygenesis: Radioactive tracers incorporated into oxygen atom in water gave radioactivity in evolved oxygen. The timescale of oxygen evolution reaction was found to be in millisecond ranges (from various sources, it was found to be anywhere from 2 to 5 milliseconds). That means that the (pseudo-first order) rate of water photolysis is $10^{2}$ to $10^{3} \mathrm{~s}^{-1}$. The oxygen evolution pattern obtained by the exposure of dark-adapted chloroplasts (primed with a "zero" time illumination) to a series of flashes of light showed a "quartet-periodicity" (before the profile attained a final steady state value). These observations (first reported by Joliot and purportedly explained by Kok) went on to become the paradigm called Joliot-Kok or Kok-Joliot "linear 4step" model, which is captured in Figure 4 (along with the essential events/elements of the ETC on the left).

Figure 4: A schematic comparison for the electron transport and oxygenesis cycles. The progression is clockwise, starting from top-left. In the ETC, through a cascade of steps (shown in the left panel with numbers 1 through 4, which can also be equated to four consecutive flashes of/within a Joliot-Kok cycle), water loses four electrons, and each electron passes via the three phases and two zaps (one each at PS I and PS II). Protons could be released at any moment of the cycle. In the oxygenesis scheme, four flashes are required from SO state to come to S4 state, leading to the liberation of oxygen. In the starting state, only the SO and S1 populations of WSCs are believed to be stable, and are supposed to exist at a ratio of 1:3. After $\sim 4$ to 6 cycles, the steady state is attained, where all four WSC populations are at a ratio of 1:1:1:1. Therefore, the starting SO state can receive 4 flashes (within a cycle) whereas the starting S1, S2 and S3 states can only receive 3, 2 and 1 flashes respectively. The criss-crossing straight lines show putative double hits, leading to two electron transitions. Triple/quadruple hits are not considered relevant.

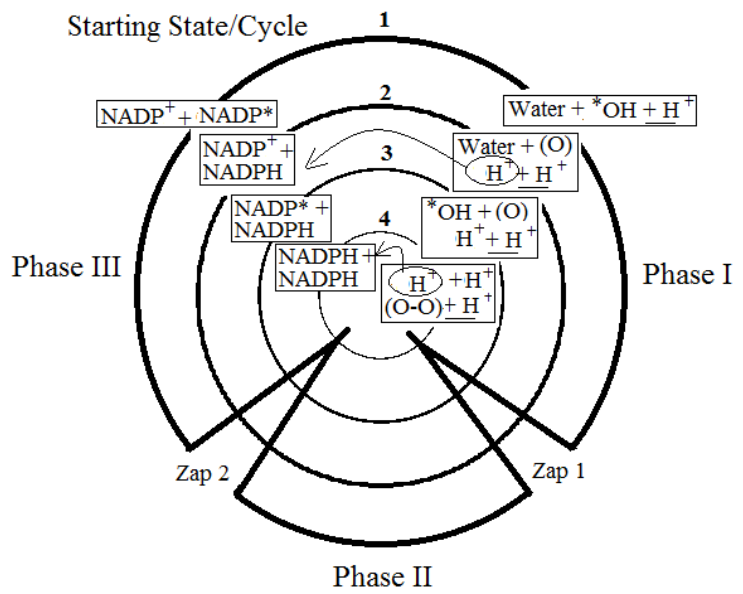

Theoretical ETC

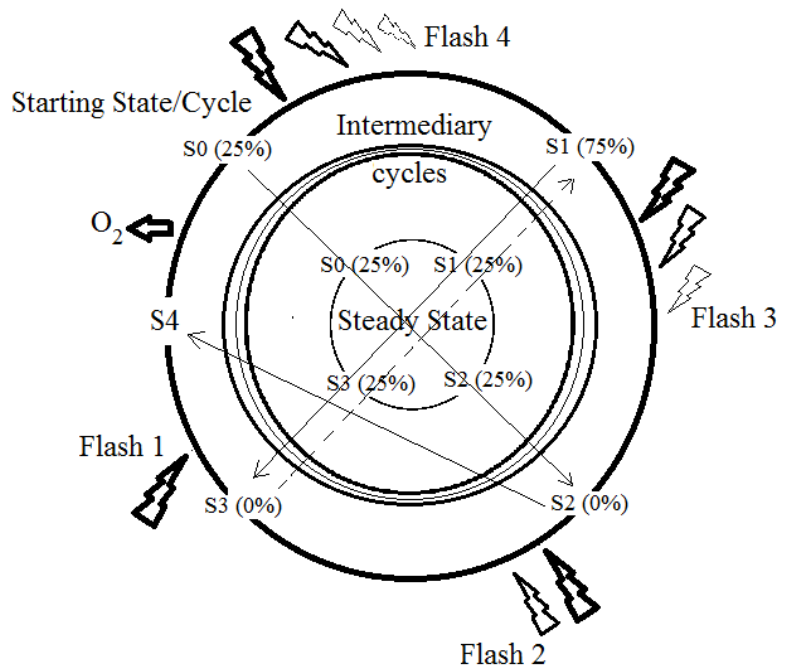

Joliot-Kok Oxygenesis

These observations, in conjunction with the supposition of the " $Z$ scheme of ETC" led to a proposal in which the WSC proceeds through a cyclic change of oxidation states from $\mathrm{S}(0)$ to $\mathrm{S}(+1), \mathrm{S}(+2), \mathrm{S}(+3)$; and transiently via $\mathrm{S}(+4)$, after which it releases oxygen, to regain the "native" $\mathrm{S}(0)$. Per the Kok-Joliot model, this linear/circular progression through the various oxidation states from 0 to +4 completes one cycle in a time frame of approximately $10^{-3}$ seconds and is supposedly mediated by a cooperativity of charges within the oxygen forming complex (wherein the four $\mathrm{Mn}$ atoms are also aided by the $\mathrm{Ca}-\mathrm{O}-\mathrm{Cl}$ atoms within). The most important aspect to be noted is that while electrons and protons can be released at various (stipulated) time 
points from the MnComplex, the molecule of oxygen is released only at one instant in the mechanistic cycle. That is- the system does not permit oxygen release in any state of the complex other than between $S(+4)$ and $S(0)$. (This supposition also implies directly that the experimental findings of DROS within the system are the products of unwanted side-reactions.) The prevailing understanding of the overall reaction mechanism solicits an "oxygen evolution center", also known as "water splitting complex" as the center stage. This role is supposedly served by the MnQuad-Ca-O-Cl cluster (henceforth called MnComplex) accompanying PS II. At this juncture, it must be seen that release of a molecule of oxygen entails the breaking of two molecules of water. Overall, this is a 4-electron reaction and all these four electrons are to be passed through into the ETC for the formation of $2 \mathrm{NADPH}$ molecules at the final stage. As per the prevailing scheme, it is the MnComplex that gives four electrons to ETC via an essential tyrosine residue at one end; and duly gets them back from two water molecules bound at alternative sites. Therefore, such an explanation solicits that the catalytic cycle of "oxygen evolution center" or "water splitting complex" holds tight at least two oxygen atoms of two molecules of water. (This is quite similar to the mOxPhos paradigm, where Complex IV holds a molecule of oxygen until 4 protons and electrons each are made available.)

In a nutshell, PS II-ensemble breaks water (liberating oxygen) to provide electrons and pumps the stromal protons into thylakoid lumen. Now, PS I-ensemble deal with the electrons, packing them into NADPH and ATP(synth)ase complex uses the lumen protons to make ATP. An electron from water needs to be powered by two photons to travel all the way to NADP ${ }^{+}$.

\subsubsection{Inhibition of $\mathrm{Pl}-\mathrm{Pp}$}

Approximately, $30 \%$ of the commercial herbicides fall under the category of Pl-Pp inhibitors. In the context, the inhibitors can be divided into two categories- those that affect PSI and those that affect PSII. The physiological symptoms are only seen if plants are illuminated (that is- the inhibitors wreak havoc by disrupting the ETC mechanism against the plant itself!) and is found to lead to chlorosis and necrosis, ultimately resulting in plant death. The carotenoids and xanthophylls present in the chloroplasts are also supposed to protect chlorophylls from photooxidation. [The sources for information within this section are Devine et al., 1993; Ahrens, 1994; Anderson, 1996; Ross \& Lembi, 1999.]

The PSI inhibitors (called membrane disruptors, electron interceptors, contact herbicides, etc.) supposedly mess up the ETC by diverting electrons from key Fe-S proteins $\left(\mathrm{F}_{\mathrm{A}} \mathrm{F}_{\mathrm{B}}\right)$ and are represented by bipyridyliums like paraquat (methyl viologen). The molecule per se is not deemed toxic but it is supposed to lead to the production of DROS, which are deemed to lead to cellular disruption.

The PSII inhibitors (represented by triazines, substituted ureas and uracils, phenyl- carbamates, nitriles, pyridazines, pyridazinones, acid amides, benzothiadiazoles, etc.) are supposed to bind to the thylakoid D1 $\mathrm{Q}_{\mathrm{B}}$ protein, thereby blocking electron transfers to the mobile plastoquinone pool.

There is yet another physical mode of inhibition, called photoinhibition, which usually results from blue light-induced damage to oxygen-evolving complex accompanying PSII. The mechanism of damage supposedly involves DROS. 
Besides these well-studied inhibitions of Pl-Pp, there are also reports of inhibitions sponsored by histones and polycations, reminiscent of the inhibition of mOxPhos ETC (Brand et al., 1971).

\subsection{Functional energetics}

The sunlight aided photosynthetic reaction occurring in chloroplasts can be minimally represented by: $\mathrm{CO}_{2}+\mathrm{H}_{2} \mathrm{O} \rightarrow \mathrm{CH}_{2} \mathrm{O}+\mathrm{O}_{2}$. The energy requirement for this reaction can be calculated from three inter-connected perspectives: bond energies, redox chemistry or thermodynamics of the overall reaction. Regardless of the approach, the theoretical requisites from all these angles run down to $\sim 500 \mathrm{~kJ} / \mathrm{mole}$. Depending on the wavelength of light within the visible spectrum $(300-170 \mathrm{~kJ} / \mathrm{mol}$ for the low and high wavelength ranges), this translates to a conservative requirement of 2-3 powering photons.

Across the visible spectrum, low absorption directly correlates to reduced photosynthetic rates. Approaching $700 \mathrm{~nm}$ (and beyond the same, wherein the chloroplasts show very little absorption), both the rate and efficiency drop to practically a zero value. The green light range $(495-575 \mathrm{~nm})$ gives low absorption and photosynthetic rates, concomitant with a relatively lesser quantum yield. It is interesting to note that quantum yield retains a $>75 \%$ of maximal value across the visual spectrum, falling sharply at the red light range, starting at $675 \mathrm{~nm}$.

It has been estimated that most natural photosynthetic systems work holistically (conversion to total plant biomass with respect the total exposure to incident sunlight energy) at anywhere between 0.1 to $2 \%$ efficiency. If we were to discount for various types of losses at the input stage, some efficient plants (taken in optimal experimental setups) were found to give up to 6-8\% efficiency for the overall photosynthetic process/conversion. It is generally believed that plant cells have very high efficiency for the relay of photons (and their energy) absorbed. Apparently, plant cells employ quantum computations to achieve this effect (Engel et al., 2007; Biello, 2007).

\section{Critiquing the standing explanation for PI-Pp}

Before coming to the arguments that are unique to Pl-Pp, a summation of the arguments against RCPE (which are relevant for both mOxPhos and Pl-Pp) would be dealt first. This exercise would serve to consolidate the cynical mindset of the reader, readying one for the radical proposal to be floated thereafter. (While dealing with experimental data, fluorescence and luminescence based experiements and inferences thereof have been not been given significance because of the consideration that such experiments would be very "sensitive" to the ROS dynamics and therefore, they are subject to a high level of uncertainty.)

\subsection{Little evidence or scope for proton pumping machineries and chemiosmotic principles:}

Historially, Peter Mitchell's hypothesis (Mitchell, 1961) and inferences from Jagendorf's observations (Jagendorf \& Uribe, 1969) held the roost in this area. These ideas have been debunked recently (Manoj, 2017; Manoj, 2018). There is nothing in the structures of phtosystem II or Cyt $b f$ (the two supposed proton pumping machineries within the Pl-Pp system) which supports the notion that proton pumps exist on thylakoid membranes. Proton availability has been pointed out as an insurmountable constraint in mOxPhos and there is little reason why it 
should not be so in chloroplasts too. Though many researchers may believe that the $\mathrm{pH}$ of thylakoid lumen is very low, we have still not developed any direct probe to accurately determine the $\mathrm{pH}$ within thylakoid lumen or stroma (both of which are nm-scaled aqueous domains) of chloroplasts. Such beliefs on the existence of proton pumps could have resulted out of the "mandate" imposed with the earlier doctrines. In spite of the concrete arguments and evidence against the "proton pumps" hypothesis, one is still obliged to indulge it because it was the Pl-Pp system that established the absolutely untenable "proton pump" concept.

Gauging from electron micrographs, an average thylakoid is $\sim 0.5$ micron in linear dimension, and the lumen is $\sim 0.05$ micron thick. Therefore, assuming a flattened (cubic or cylindrical) vesicular structure, the maximal volume of a thylakoid would be $\sim 10^{-17}$ liters. As a result, in the starting or resting phase, the protons within the lumen of thylakoid (at $\mathrm{pH} 7$ ) would be-

$6.023 \times 10^{23} \times 10^{-7} \times 10^{-17} \leq 10^{0}$

Therefore, the thylakoid is practically aprotic! (This does not mean that protons are not available for catalysis. Grotthuss mechanism would ensure catalytic process but pumping protons out into another and discontinuous macrosystem is a totally different game.) The stroma would have a much higher volume (say, $5 \mu \mathrm{m} \times 5 \mu \mathrm{m} \times 5 \mu \mathrm{m}$ ) of $\sim 10^{-13}$ liters, and it would have protons approaching a total of-

$6.023 \times 10^{23} \times 10^{-7} \times 10^{-13} \leq 10^{4}$

(The reader should not get confused that these difference in numbers could lead to gradients. The concentration in the two phases are still the same!) Assuming an initial closed system of 100 thylakoids within a chloroplast (at $\mathrm{t} 1$ ), only if the protein complexes efficiently pumped $>90 \%$ of the stromal protons into the thylakoids (at $\mathrm{t} 2$ ), the gradient across the thylakoid membrane would approach the energy term capable of ATP synthesis. [This is given by: $61 \log \{90 /(0.5 \times 0.5 \times$ $0.05)\} /\{1000 /(5 \times 5 \times 5)\}=180 \mathrm{mV}$. Here, the volume term of a thylakoid is $0.01 \%$ of the stroma. Even if the thylakoids are connected (as we know they are; please refer Figure 4 from the site- http://teachers.yale.edu/curriculum/search/viewer.php?skin=h\&id=initiative_07.05.08_u), we would have the same calculation because while the total lumen volume increases by 100 folds, the proton amount in the lumen also gets multiplied by 100. It is known that 180 to 200 $\mathrm{mV}$ (correlating to a concentration gradient of 900 folds!) is the minimal theoretical or practical requirement for deriving or observing ATP synthesis, per the "transmembrane pump" mechanistic purview (Nicholls, 2004).]

The calculation above is from an initial state to a final state and it cannot explain the steady state functioning of the chloroplast. Since the pumps are not synchronized and there are no modularities (that is- a protein pump is not associated with its own "outer shell", and several such shells are not triggered simultaneously with some phase transition operation), the proton concentration would rather equilibrate in steady state, than create a gradient. A simple quantitative purview is enough to dismiss the vanity of "proton pump" concept for the thylakoid membrane- When Grotthuss mechanism would ensure proton availability to a given "proton pumping protein", why should there be thousands of proteins to deal with a few decades of protons? Also, how can the proteins flip back and forth into different conformations under a 
steady state trans-membrane potentials (which they themselves set up)? Quite simply, in the absence of any direct evidence for proton pumps, all these impossible requirements seek too much intelligence at the proteins' ends. Let us not forget that there is little thermodynamic logic in assuming that the protons would "have any urge" to spontaneously return to the stroma (Manoj 2017). For further discussions in this regard, please refer the follow-through communication discrediting the RCPE explanation for mOxPhos (Manoj 2018).

\subsection{Evolutionary perspectives and structure-function correlations:}

* Once the "Proton pump- Chemiosmotic mechanism" principle was shown to be a fallacy, the elaborate ETC scheme of Pl-Pp does not have any evolutionary or functional significance. That is- Why would nature evolve such an ETC that doesn't serve any significant function? Say, why did the PS II not relay the electrons from water directly to $\mathrm{Fd} / \mathrm{NR}$ ?

* It is natural to envisage that primordial living beings could have possessed a spontaneous power generating system, without the "gambit" logic that the debunked proton pump cum chemiosmosis mechanism supposes. All "evolutionary logic" arguments stated against the RPCE explanation (like "non-evolvability due to irreducible complexity" for mOxPhos are relevant herein too (Manoj, 2017; Manoj, 2018). It is improbable that a vitally deterministic scheme could evolve downplaying the inherent reactivity of the components employed within the system. Why should the cellular system choose a lesser efficient system when substrate-level oxidation gives more efficiency (and that too, without the threat of forming DROS)? It is inconceivable how Complex V could function reversibly in situ (with the exact same mechanism) when it has higher affinities for ATP (in comparison to ADP) (Manoj, 2018; Manoj et al., 2018).

* Why do leaves that show high rates of photosynthesis have low life spans (Matsuiki \& Koike, 2006)? This reality would be highly counter-intuitive and counter-evolutionary!

* Structural information available leaves little scope for modularized synchronization or transmembrane potential (TMP) tapping mechanisms for the various structure-function components to work in tandem (with respect to ETC, Proton pumps, Chemiosmotic mechanism and Rotary ATP synthesis). The chloroplast is an oxygenic system. Oxygen (singlet/triplet) and DROS are spontaneously formed in chloroplasts. It is impossible that any electronic circuitry or redox catalytic/relay mechanism could evolve without oxygen/DROS messing up with the "vitally deterministic mechanisms" (Manoj, 2017; Manoj, 2018). Structural elucidations have not demonstrated any specific oxygen evolution routes within PS II for a "safe-release" outside the chloroplast. In fact, the fact that most PS II systems are seated deep within the thylakoid stacks is antithetical to the hitherto attributed structure-function correlations.

* The structure of a chloroplast with varying numbers and stories/stacks of thylakoids (as seen from the writing and images of Section 2.1), with a random distribution of the thylakoid proteins and pigments, often showing significant variations based on the particular species, locations within the leaf, durations of development, etc. do not speak of a "deterministic vital scheme" that the EPCR explanation solicits. Particularly- (a) From the PS II centers located deep within the multi-layered stacks of thylakoids, it is inconceivable how the Cyt $b f$ - plastocyanin system could relay an electron in any feasible time to the outer stack-located PS I centers for explaining the 
"Emerson effect". (b) If the above point (a) is true, then the deep-seated PS II systems cannot even work in conjunction with the PS I center (in a "non-concerted setup where PS I is blocked, say), they can't even work independently. Surely, it would be even more difficult to get the electrons relayed to the Ferredoxin-NADP reductase system present within the stroma. So, why should there be multi-storied stacks of thylakoids (when it is more "sensible" for evolution to have one-three layers, at the most, for efficient electron transfers between the various Complexes)?

* The distances between the redox centers and distributions of the elements of the vitally deterministic ETC leave a lot desired with respect to factual viability. The structures of PS I and PS II (for example: Fromme et al., 2001; Young et al., 2016; Nelson \& Ben-Shem, 2004; Nelson \& Yocum, 2006) show that many times, the distances between key redox centers are above 12 Angstroms (the crucial distance that could give a viable electron transfer rate). Further, there are several other redox active species that are located (much closer by) within transerable distance. If Marcus theory was the fundamental operative logic, it is quite unclear as to why the closer located species could not shunt the proposed ETC routes! The arrangement of redox potentials are also not in the strict ascending gradient, as exemplified by the $\mathrm{F}_{\mathrm{A}}$ to $\mathrm{F}_{\mathrm{B}}$ purported transfer in PS I leg of the ETC. (Please refer Manoj, 2018 for the details in the critiquing logic targeted against the "vitally deterministic ETC of mOxPhos". The same arguments/analyses are relevant here too.)

The arguments stated above seek a forthright and critical overhaul of the $\mathrm{Z}$ scheme and JoliotKok cycle of Pl-Pp.

\subsection{Deconstructing the multi-step Z-scheme for Pl-Pp ETC and 4-step Joliot-Kok cycle of oxygen evolution at WSC:}

To begin with (just like the problem with the naming of the concept of chemiosmosis), there is the question of semantics of calling the prevailing Pl-Pp ETC a Z scheme. It is evident from Figure 3 that there are three downhill ET phases and two uphill zaps. Therefore, ideally, it should be called an "overlapped double-Z or zig-zag scheme" or better, a "thunderbolt scheme", correlating to the way a thunderbolt is drawn with the rollercoaster route an electron from water gets projected through its journey, all the way to forming NADPH!

Let us set an electron relay into motion by the excitation of ChlP680 with a photon of suitable wavelength. (That is- we operate Zap 1 alone.) At the end of a suitable time, let us assume that one electron would have traveled through Phases I \& II (without actually going all the way into the final acceptor of $\mathrm{ChlP} 700^{+}$). Repeated operation of Zap 1 would not enable any further acyclic electron transfers because Phase II cannot be completed and electron(s) cannot get transferred to $\mathrm{ChlP} 700^{+}$(because Zap 2 has not occurred) and thereafter onwards to NADP ${ }^{+}$. On the other hand, if we had operated Zap 2, one electron would have only completed Phase III. Repeated operation of Zap 2 would not lead to any further relay even in this phase because the oxidized ChlP700 ${ }^{+}$cannot get excited or give electrons any further. So, we see that for the prevailing $\mathrm{Z}$ scheme to be operative (for the complete one-electron travel all the way from water to $\mathrm{NADP}^{+}$, completing all three phases), two zaps would be required (one photon each would be required for zapping each one of the photosystems) for overcoming the breaks in continuity. 
These two "circuit-breaking" discharge-charge sequences at the photosystems (namely: ChlP680* - e $\rightarrow$ ChlP680 + e $\rightarrow$ ChlP680 and ChlP700* - e $\rightarrow$ ChlP700 $^{+}+$e $\rightarrow$ ChlP700; please refer Figure 2) arise because-

1. Electron relay from water cannot charge an electron-satisfied ChlP680 and/or an electron deficient ChlP680 $0^{+}$cannot get photo-excited to ChlP680*. (Let's call this as Gap 1.)

2. ChlP680* cannot transfer electrons to an electron-satisfied ChlP700 and/or an electrondeficient ChlP700 ${ }^{+}$cannot get photo-excited to ChlP700*. (Let's call this as Gap 2.)

Please see that we have to write both the oxidized and reduced forms of the two key ETC/photoexcitable components (ChlP680 \& ChlP700) in the diagram (Figure 3) as a prerequisite for the three ETC phases and the two photon zaps to work repeatedly! Therefore, since this is a disrupted sequential acyclic process, the elements cannot be given the same representation as routine electron transfer chains. [[A comparison is in order, to elicit the point. For example- the purported mOxPhos ETC relay- "NADPH $\rightarrow$ Complex I $\rightarrow$ CoQ $\rightarrow$ Complex III $\rightarrow$ Cyt c $\rightarrow$ Complex IV $\rightarrow$ Oxygen" would not have such breaks. This is because the primary source of electrons (NADH) is taken in the reduced state and all the subsequent elements of the ETC (including the final acceptor of electrons of oxygen) are taken to be in the oxidized state. This is the requisite for a continuous downhill electron transfer process. In the mOxPhos system, two NADH molecules could give two electrons each to two oxidized Complex I molecules, which would reduce the latter. The reduced Complex I molecules would now give the four electrons to two CoQ molecules and reduce them, while reverting back to the oxidized state. This process would go all the way until the last element of the ETC where molecular oxygen ultimately accepts the electrons to and forms water. Thus, the given elements of the ETC can "theoretically" sustain the electron relay by continuously shifting redox states upon receiving and giving one or two electrons. One can see that the requirement for depicting such feasibility is that all elements (except the primary electron donor) within the relay must be oxidized at the commencement of the ETC. If we do the same exercise for the Pl-Pp ETC, we need to write ChlP680 ${ }^{+}$as the terminal electron acceptor for Phase I. But if we do so, it will not allow us to incorporate the photo-excitability element of Pl-Pp into the scheme any further.]] Therefore, the more "theoretically correct" Pl-Pp scheme (as shown in Figure 3) enables us to visualize how the purported Z scheme affects the feasibility of the overall process. Now, the sequence of ET events boils down to two series of concomitant and distinct/discrete but interdependent (and repeated) temporal electron transfer operations centered at the two loci (PS II and PS I), as captured in Figure 5 below. The spatio-temporal essence of the same is captured by two operations.

Operation 1 (Overall uphill steps), Zap \& Discharge: (i) P680* to PC (Zap I \& Phase II) AND (iii) P700* to NADP ${ }^{+}$(Zap II \& Phase III)

$+$

Operation 2 (Overall downhill steps), Charge: (iia) $\mathrm{PC}^{*}$ to $\mathrm{P} 700^{+}$(Charge II) AND (iib) Water to $\mathrm{P} 60^{+}$(Phase I and Charge I)

Figure 5: A breakdown of the electron transfer phenomenology within the purported " $Z$ scheme" of Pl-Pp ETC, with a spatio-temporal perspective. The transfer of electrons from stable (reduced) participants to yield stable (reduced) products within a reaction leg is shown. Since the reaction turnover is in millisecond timescale, stability is defined as any time period that is significantly comparable to this scale. 


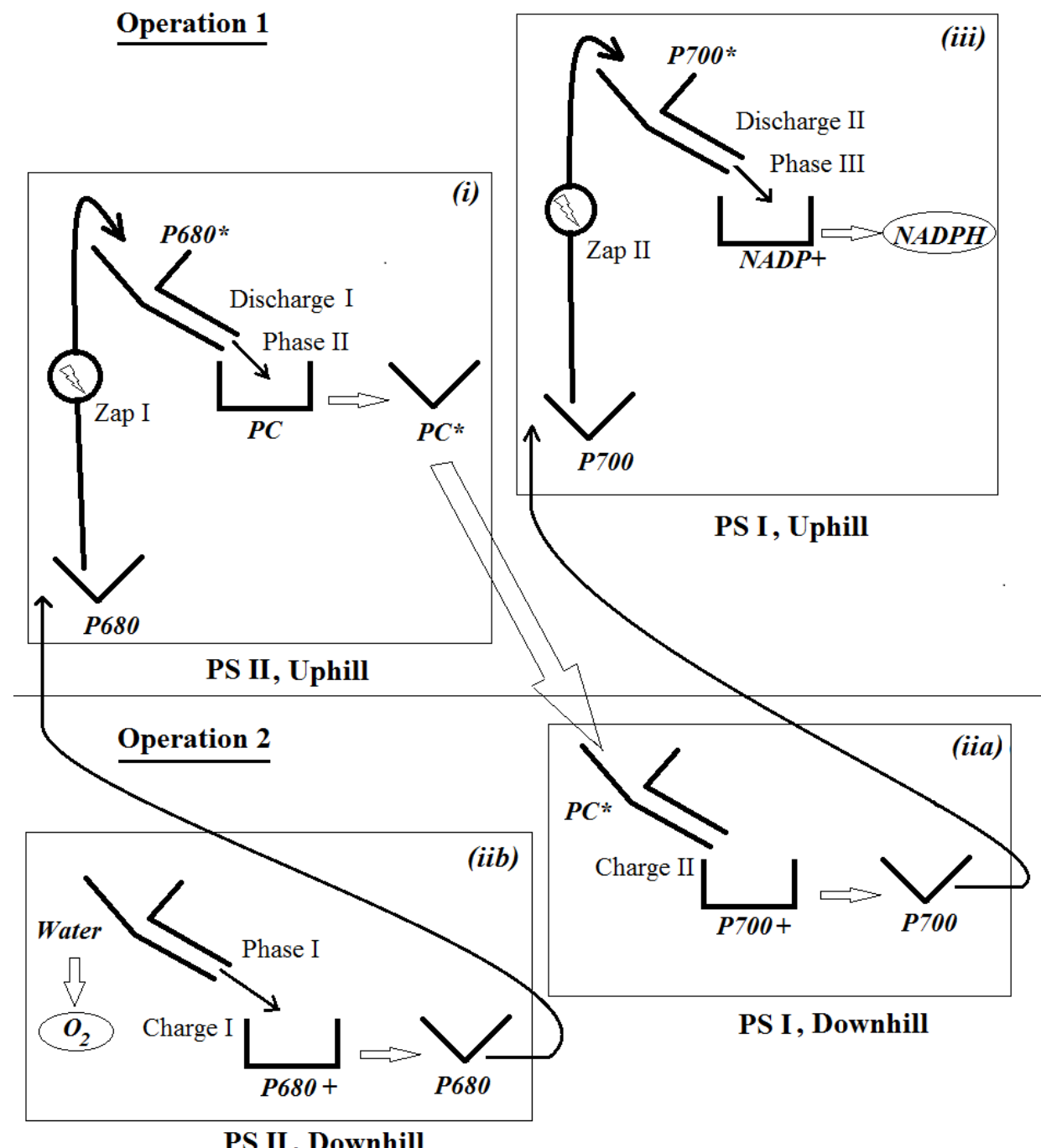

Now, for the evolution of a molecule of oxygen, the setup must go through the precise sequential and iterated orchestrations of 4 cycles: Operation $\mathrm{p} 1+$ Operation $\mathrm{p} 2+$ Operation $\mathrm{q} 1+$ Operation $\mathrm{q} 2+$ Operation $\mathrm{r} 1+$ Operation $\mathrm{r} 2+$ Operation $\mathrm{s} 1+$ Operation s2 steps; where $\mathrm{p}, \mathrm{q}, \mathrm{r}$ and $\mathrm{s}$ are sequential rounds of one-electron equivalents (totaling four) entering the ETC. Very clearly, the most crucial "bottleneck" step in the overall acyclic sequence is the dynamics of Phase II, leading to the production and transport of $\mathrm{PC}^{*}$, which ultimately governs the connectivity of operations between the spatially disconnected reaction centers of PS II and PS I. (This step is akin to cytochrome $c$ serving 4 electrons to Complex IV, in the mOxPhos system. Such mechanistic processes were shown to be untenable (Manoj, 2018 ;Manoj et al., 2018).

Now, for synchronizing ETC and oxygenesis cycles (as extended from Figures $3 \& 4$ and Table 1), let us ideate a simple and favorable case scenario. Let it be given that a chloroplast has 8 molecules of water bound to 4 WSC units and let there be 4 molecules each of ChlP680 and ChlP700 (along with an unlimiting amount of the required/optimal redox-relay intermediaries and final acceptor molecule, $\mathrm{NADP}^{+}$). Let us now suppose that they are arranged in an optimally well-knit circuit and let us also agree that these elements and their various redox forms are stable 
at the starting point and through the various steps of the cycle. Let us now assume that both push-pull mechanisms of electron transfer are operative. These ideas are represented within Table 1. The details shown therein are discussed in the two paragraphs below.

Now, disregarding the time-frame needs for several zapping, electron-conduction and transport requirements, let us take an account of the overall process. There are $>20$ redox active species in the $\mathrm{Z}$ scheme with approximately same amount of distinct intra- or inter- molecular electron transfer steps (from $\mathrm{H}_{2} \mathrm{O}$ to $\mathrm{NADP}^{+}$). Each species can be in oxidized or reduced state and therefore, the chance of finding the particular relay agent in the suitable redox state is $1 / 2$. So, the sequential scheme's overall crude score (within all available sample space) would be $(1 / 2)^{20}$ or less than one in 33 million! [Introducing complexities into this analysis is an exercise in vanity but we shall indulge in it, albeit briefly. Let's just consider the four key components of the ETC, "WSC-PS II-PS I-2NADP",. With respect to the bound ligands and without considering symmetry aspects, a WSC can exist in a total of 5 states $\left(\mathrm{H}_{2} \mathrm{O}-\mathrm{H}_{2} \mathrm{O}, \mathrm{H}_{2} \mathrm{O}-\mathrm{OH} *, \mathrm{H}_{2} \mathrm{O}-\mathrm{O},{ }^{*} \mathrm{HO}-\mathrm{O}\right.$, $\mathrm{O}-\mathrm{O})$ of which four can favor the reaction. Each one of the two photosystems can be in three unequal states- native, excited and discharged, of which only one can favor the reaction. Similarly, the final leg can also be in three states: $\mathrm{NADP}^{+}, \mathrm{NADP}^{*}$ and NADPH, and out of these, two can support the reaction. Now, the fruitful reaction of the sequentially wired "WSCPS II-PS I-2NADP ${ }^{+}$" system would be: $(4 / 5) \times(1 / 3) \times(1 / 3) \times(2 / 3)=8 / 135$ or $<(1 / 2)^{4}$. Now, if we factor in the remaining elements of $\sim 16$ redox species (with each one of these transients having an oxidized and a reduced state), the probability of favorable outcome within the overall sample space would be $(1 / 2)^{16} \times 8 / 135, \approx(1 / 2)^{20}$ ! If we consider that intricately connected redox centers within a particular protein are not subject to such probability calculations, that still makes the "Water-WSC-P680-Ph-Q ${ }_{\mathrm{A}}-\mathrm{Q}_{\mathrm{B}}-$ Cyt.bf-PC-P700-- $\mathrm{A}_{0}-\mathrm{A}_{1}-\mathrm{FeS}-\mathrm{Fd}-\mathrm{NR}-\mathrm{NADP}$ ", $\quad$ serial connectivity a formidable 15-component relay. This would still have a highly fastidious probability score of $(1 / 2)^{15}$ or less than one in a million!]

In a "minimally realistic" scenario with the 4 PS I + 4 PS II system setup, as simulated in Table 1 , the chances of getting synchronized reactions are of very low probability. If we buy the Kokreasoned scenario that three out of four WSC centres are in the native state and one is in a partially excited state (at T0 time), then we need to have all the remaining centers go through the precisely coordinated scheme of events with each zap, discharge and recharge routine. To beat the insurmountable probabilistic odds, this scheme would require some immaculate connectivity/wiring and ordered locations for the participants. However, neither the process of morphological differentiation of leaf tissue nor the final structures of various leaves show any such anatomical or structural evidence.

Table 1. Idealized temporal phasing of intermediates and electron flow through various components of the Z-scheme vis a vis Kok-Joliot oxygen evolution cycle: A minimalist and stoichiometrically idealized model for the first four flashes (leading to a 16-electron reaction) in a chloroplast with four modules each of PS I and PS II. This scheme has been visualized starting from Kok's proposed ratio of S1:S0 = 3:1 (wherein at the initial state, $S O+S 1=100 \% ; S 2=S 3=0 \%$ ). Discounting misses and double-hits, the $3^{\text {rd }}$ and $4^{\text {th }}$ Zaps' oxygen evolution corresponds to initial $S 1$ and SO populations, respectively. The process herein would be represented by the schema in Figure 3. 


\begin{tabular}{|c|c|c|c|c|c|}
\hline Cycle No. & State & WSC Status & PS II Status & PS I Status & Products \\
\hline & T0 Rest & $\begin{array}{l}\mathrm{H}_{2} \mathrm{O}-\mathrm{OH}^{*}, \quad \mathrm{H}_{2} \mathrm{O}- \\
\mathrm{OH}^{*}, \quad \mathrm{H}_{2} \mathrm{O}-\mathrm{OH}^{*}, \\
\mathrm{H}_{2} \mathrm{O}-\mathrm{H}_{2} \mathrm{O}\end{array}$ & $\begin{array}{l}\text { P680, P680, } \\
\text { P680, P680 }\end{array}$ & $\begin{array}{l}\text { P700, P700, } \\
\text { P700, P700 }\end{array}$ & Nil \\
\hline \multirow{2}{*}{$\begin{array}{l}\text { Operation } \\
\quad p 1\end{array}$} & $T 11^{\text {st }}$ Zap & $\begin{array}{l}\mathrm{H}_{2} \mathrm{O}-\mathrm{OH} *, \quad \mathrm{H}_{2} \mathrm{O}- \\
\mathrm{OH}^{*}, \quad \mathrm{H}_{2} \mathrm{O}-\mathrm{OH}^{*}, \\
\mathrm{H}_{2} \mathrm{O}-\mathrm{H}_{2} \mathrm{O}\end{array}$ & 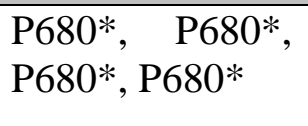 & $\begin{array}{l}\mathrm{P} 700 *, \quad \mathrm{P} 700 * \\
\mathrm{P} 700 *, \mathrm{P} 700 *\end{array}$ & Nil \\
\hline & $\begin{array}{l}\text { T1 } \\
\text { Discharge }\end{array}$ & $\begin{array}{l}\mathrm{H}_{2} \mathrm{O}-\mathrm{OH} *, \quad \mathrm{H}_{2} \mathrm{O}- \\
\mathrm{OH}^{*}, \quad \mathrm{H}_{2} \mathrm{O}-\mathrm{OH}^{*}, \\
\mathrm{H}_{2} \mathrm{O}-\mathrm{H}_{2} \mathrm{O}\end{array}$ & $\begin{array}{l}\mathrm{P}_{680^{+}}, \quad \mathrm{P}^{6} 80^{+} \\
\mathrm{P}_{680^{+}}, \mathrm{P}^{+}\end{array}$ & $\begin{array}{l}\mathrm{P} 700^{+}, \quad \mathrm{P} 700^{+} \\
\mathrm{P}^{+} 00^{+}, \mathrm{P}^{+} 00^{+}\end{array}$ & 2NADPH \\
\hline $\begin{array}{l}\text { Operation } \\
\text { p2 }\end{array}$ & $\begin{array}{l}\text { T1 } \\
1^{\text {st }} \text { Charge }\end{array}$ & $\begin{array}{l}\mathrm{H}_{2} \mathrm{O}-\mathrm{O}, \quad \mathrm{H}_{2} \mathrm{O}-\mathrm{O}, \\
\mathrm{H}_{2} \mathrm{O}-\mathrm{O}, \mathrm{H}_{2} \mathrm{O}-\mathrm{OH}^{*}\end{array}$ & $\begin{array}{l}\text { P680, P680, } \\
\text { P680, P680 }\end{array}$ & $\begin{array}{l}\text { P700, P700, } \\
\text { P700, P700 }\end{array}$ & $\left(2 \mathrm{H}^{+}\right)$ \\
\hline \multirow{2}{*}{$\begin{array}{l}\text { Operation } \\
q 1\end{array}$} & $T 22^{\text {nd }}$ Zap & $\begin{array}{l}\mathrm{H}_{2} \mathrm{O}-\mathrm{O}, \quad \mathrm{H}_{2} \mathrm{O}-\mathrm{O} \\
\mathrm{H}_{2} \mathrm{O}-\mathrm{O}, \mathrm{H}_{2} \mathrm{O}-\mathrm{OH}^{*}\end{array}$ & $\begin{array}{l}\mathrm{P} 680^{*}, \quad \mathrm{P} 680^{*}, \\
\mathrm{P} 680^{*}, \mathrm{P} 680^{*}\end{array}$ & $\begin{array}{l}\mathrm{P} 700^{*}, \quad \mathrm{P} 700^{*}, \\
\mathrm{P} 700^{*}, \mathrm{P} 700^{*}\end{array}$ & Nil \\
\hline & $\begin{array}{l}\text { T2 } \\
\text { Discharge }\end{array}$ & $\begin{array}{l}\mathrm{H}_{2} \mathrm{O}-\mathrm{O}, \quad \mathrm{H}_{2} \mathrm{O}-\mathrm{O}, \\
\mathrm{H}_{2} \mathrm{O}-\mathrm{O}, \mathrm{H}_{2} \mathrm{O}-\mathrm{OH}^{*}\end{array}$ & $\begin{array}{l}\mathrm{P}_{680}{ }^{+}, \\
\mathrm{P} 680^{+}, \\
\mathrm{P} 680^{+}\end{array}$ & $\begin{array}{l}\mathrm{P} 700^{+}, \quad \mathrm{P}^{+} 00^{+}, \\
\mathrm{P}^{+} 00^{+}, \mathrm{P}^{+} 00^{+}\end{array}$ & 2NADPH \\
\hline $\begin{array}{l}\text { Operation } \\
q 2\end{array}$ & $\begin{array}{l}T 2 \\
2^{\text {nd }} \text { Charge }\end{array}$ & $\begin{array}{l}* \mathrm{HO}-\mathrm{O}, \quad * \mathrm{HO}-\mathrm{O}, \\
* \mathrm{HO}-\mathrm{O}, \mathrm{H}_{2} \mathrm{O}-\mathrm{O}\end{array}$ & $\begin{array}{l}\text { P680, P680, } \\
\text { P680, P680 }\end{array}$ & $\begin{array}{l}\text { P700, P700, } \\
\text { P700, P700 }\end{array}$ & $\left(2 \mathrm{H}^{+}\right)$ \\
\hline \multirow{2}{*}{$\begin{array}{c}\text { Operation } \\
\quad r l\end{array}$} & $T 33^{\text {rd }}$ Zap & $\begin{array}{l}* \mathrm{HO}-\mathrm{O}, \quad * \mathrm{HO}-\mathrm{O}, \\
* \mathrm{HO}-\mathrm{O}, \mathrm{H}_{2} \mathrm{O}-\mathrm{O}\end{array}$ & $\begin{array}{l}\text { P680*, } \mathrm{P} 680 * \\
\text { P680*, P680* }\end{array}$ & $\begin{array}{l}\mathrm{P} 700^{*}, \quad \mathrm{P} 700^{*}, \\
\mathrm{P} 700^{*}, \mathrm{P} 700^{*}\end{array}$ & Nil \\
\hline & $\begin{array}{ll}\text { T3 } & 3^{\text {rd }} \\
\text { Discharge } & \end{array}$ & $\begin{array}{l}* \mathrm{HO}-\mathrm{O}, \quad * \mathrm{HO}-\mathrm{O}, \\
* \mathrm{HO}-\mathrm{O}, \mathrm{H}_{2} \mathrm{O}-\mathrm{O}\end{array}$ & $\begin{array}{l}\mathrm{P}_{680^{+}}, \quad \mathrm{P}_{680^{+}}, \\
\mathrm{P} 60^{+}, \mathrm{P}^{+}\end{array}$ & $\begin{array}{l}\mathrm{P} 700^{+}, \quad \mathrm{P}^{+} 00^{+}, \\
\mathrm{P}^{+} 00^{+}, \mathrm{P}^{+} 00^{+}\end{array}$ & 2NADPH \\
\hline $\begin{array}{l}\text { Operation } \\
\quad r 2\end{array}$ & $\begin{array}{l}\text { T3 } \\
3^{\text {rd }} \text { Charge }\end{array}$ & $\begin{array}{l}\mathrm{H}_{2} \mathrm{O}-\mathrm{H}_{2} \mathrm{O}, \quad \mathrm{H}_{2} \mathrm{O}- \\
\mathrm{H}_{2} \mathrm{O}, \quad \mathrm{H}_{2} \mathrm{O}-\mathrm{H}_{2} \mathrm{O}, \\
* \mathrm{HO}-\mathrm{O}\end{array}$ & $\begin{array}{l}\text { P680, P680, } \\
\text { P680, P680 }\end{array}$ & $\begin{array}{l}\text { P700, P700, } \\
\text { P700, P700 }\end{array}$ & $3 \mathrm{O}_{2}\left(2 \mathrm{H}^{+}\right)$ \\
\hline \multirow{2}{*}{$\begin{array}{c}\text { Operation } \\
\text { sl }\end{array}$} & $T 44^{\text {th }}$ Zap & $\begin{array}{l}\mathrm{H}_{2} \mathrm{O}-\mathrm{H}_{2} \mathrm{O}, \quad \mathrm{H}_{2} \mathrm{O}- \\
\mathrm{H}_{2} \mathrm{O}, \quad \mathrm{H}_{2} \mathrm{O}-\mathrm{H}_{2} \mathrm{O}, \\
* \mathrm{HO}-\mathrm{O}\end{array}$ & 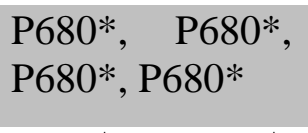 & $\begin{array}{l}\mathrm{P} 700 *, \quad \mathrm{P} 700 * \\
\mathrm{P} 700 *, \mathrm{P} 700 *\end{array}$ & Nil \\
\hline & $\begin{array}{l}\text { T4 } \\
\text { Discharge }\end{array} 4^{\text {th }}$ & $\begin{array}{l}\mathrm{H}_{2} \mathrm{O}-\mathrm{H}_{2} \mathrm{O}, \quad \mathrm{H}_{2} \mathrm{O}- \\
\mathrm{H}_{2} \mathrm{O}, \quad \mathrm{H}_{2} \mathrm{O}-\mathrm{H}_{2} \mathrm{O}, \\
* \mathrm{HO}-\mathrm{O}\end{array}$ & $\begin{array}{l}\mathrm{P}_{680^{+}}, \quad \mathrm{P}_{680^{+}}, \\
\mathrm{P}_{680^{+}}, \mathrm{P} 60^{+}\end{array}$ & $\begin{array}{l}\mathrm{P} 700^{+}, \\
\mathrm{P}^{+} 0^{+}, \mathrm{P} 700^{+}\end{array}$ & 2NADPH \\
\hline $\begin{array}{c}\text { Operation } \\
s 2\end{array}$ & $\begin{array}{l}\text { T4 } \\
4^{\text {th }} \text { Charge }\end{array}$ & $\begin{array}{l}\mathrm{H}_{2} \mathrm{O}-\mathrm{OH} *, \quad \mathrm{H}_{2} \mathrm{O}- \\
\mathrm{OH}^{*}, \quad \mathrm{H}_{2} \mathrm{O}-\mathrm{OH}^{*}, \\
\mathrm{H}_{2} \mathrm{O}-\mathrm{H}_{2} \mathrm{O}\end{array}$ & $\begin{array}{l}\text { P680, P680, } \\
\text { P680, P680 }\end{array}$ & $\begin{array}{l}\text { P700, P700, } \\
\text { P700, P700 }\end{array}$ & $\mathrm{O}_{2}\left(2 \mathrm{H}^{+}\right)$ \\
\hline 4 cycles & $4(x 2)$ zaps & $\begin{array}{l}{[4 \mathrm{WSC}]} \\
8 \mathrm{H}_{2} \mathrm{O}\end{array}$ & $\begin{array}{l}{[4 \text { ChlP680] }} \\
4 \text { photons }(\lambda 1)\end{array}$ & $\begin{array}{l}\text { [4ChlP700] } \\
4 \text { photons }(\lambda 2)\end{array}$ & $\begin{array}{l}4 \mathrm{O}_{2}, \quad 8 \mathrm{H}^{+} \\
\text {8NADPH }\end{array}$ \\
\hline
\end{tabular}

Changing the analysis by enhancing the time-frames of individual components (or by increasing the number of hits in time or even taking out a few components!) does not significantly enhance the appeal of the $\mathrm{Z}$ scheme, because the espoused mechanism is obligatorily ordered and sequential. That is, the purported scheme is $\mathrm{A}$ to $\mathrm{B}$ to $\mathrm{C}$ to $\mathrm{D} \ldots$ all the way till $\mathrm{Z}$; and not via a haphazard manner like $\mathrm{A}$ to $\mathrm{C}$ to $\mathrm{D}$ to $\mathrm{B} \ldots \mathrm{Z}$ or shunted fashion like $\mathrm{A}$ to $\mathrm{C}$ to $\mathrm{Z}$ ). Regardless, let's now get to the analysis of time. The generation of NADPH (the last reaction) is a millisecond phenomenon. Per the $\mathrm{Z}$ scheme, there are at least four reactions in the order of 0.1 to 1.0 milliseconds $\left(\mathrm{WSC} \rightarrow \mathrm{Y}_{\mathrm{Z}}, \mathrm{Q}_{\mathrm{A}} \rightarrow \mathrm{Q}_{\mathrm{B}}, \mathrm{FeS} \rightarrow \mathrm{Cyt} . f, \mathrm{Cyt} . f \rightarrow \mathrm{PC}\right.$ ) and two reactions are in the millisecond scales $\left(\mathrm{Fd} \rightarrow \mathrm{NR}, \mathrm{Q}_{\mathrm{B}} \rightarrow \mathrm{PQ}\right)$. Very curiously, the $\mathrm{Q}$ the cycle is supposed to average $\sim 10$ milliseconds. Both the splitting of water and the synthetic rate of NADPH/ATP are experimentally observed to be in the order of milliseconds. Now, once again, we have the same 
conundrum that we had in the mXM and mOxPhos mechanistic puzzle- the individual steps in a sequential scheme seem to need longer time window than the overall reaction time-frame! Quite simply, the scheme is therefore, not physiologically viable. Working with photosystems, several workers have shown that the intermediates resulting out of photoexcitation are stable for several seconds (and this is also evident by the protocols of the Kok-Joliot experiment). Then, how is it that the ETC mechanism is functional and how can it ever work in conjunction with the KokJoliot cycle? The half-lives of excited states of P700 and P680 differ significantly. When the overall ETC has millisecond duration and the electron relay is supposedly set in spontaneously at the charge of the intermediates at the top, the intermediates should not accumulate for such duration. This can only result if the downward ET processes were bottlenecks. If that were so, the operations $1 \& 2$ cannot be synchronized.

Now, let us account for the reaction components. In a chloroplast, there are $10^{2}$ thylakoids, with each thylakoid containing $10^{2}-10^{3}$ PS I / PS II and there are approximately 10 times quinones per a given PS. Now, Table 1 of a meticulous study (Haehnel et al., 1989) provides crucial information for understanding the plastocyanin distribution in chloroplasts. They found that- (i) in dark, plastocyanin is relatively more abundant in stroma, than in grana, (ii) 3 PC molecules were found per every couple of micrometers of lumen, (iii) total count of PC varied from $10^{2}$ to $10^{3}$ per chloroplast. This corresponds to a 1:1 stoichiometry of PS I and PC. The diffusion constant of PC is $\sim 10-100 \mu \mathrm{m}^{2} / \mathrm{s}$ (Sanderson et al., 1986) which implies that an average PC molecule can muster a displacement rate of $3-10 \mathrm{~nm} / \mathrm{ms}$. This dimension is in the approximate range of the linear spread of a PS system and the width of lumen or membrane. Clearly, the availability and mobility of PC would be a constraint for a millisecond time-frame reaction. Let's go to an earlier step. The electrons have to reach from the deep-seated appressed granal PS II to Cyt $b f$ via PQ to PC. If we see the experimentally calculated PQ's diffusion coefficient of 0.01 $0.3 \mathrm{\mu m}^{2} / \mathrm{s}$ within thylakoid membranes (Blackwell et al., 1994; Kirchhoff, 2014), the maximal linear displacement rate is only $0.2 \AA / \mathrm{ms}$ ! This means that PQ is practically immobile with respect to the limiting reaction time. Considering the high density of protein packing in the thylakoid membranes, the diffusion would only be more torturous and the lateral displacement rate would only be lower than the ones calculated. Though the complete reduction of PS I only takes a few decades of microseconds (and thus, is not rate-limiting), the translocation afforded by PQ and PC would become rate-limiting! Therefore, the Operation 1 \& Operation 2 synchronization cannot be achieved in the deterministic way (as espoused by Z-scheme and Joliot-Kok cycle), and therefore, elaborate Pl-Pp ETC explanation is untenable owing to the existence of multiple rate-limiting steps.

Why did one go through this elaborate presentation of the sequence of operations and states within an idealized chloroplast? It was done to understand/demonstrate the absolutely fastidious nature of the reaction scheme espoused by the prevailing paradigm! From the minimal chloroplast model (Table 1) of the "vitally deterministic Z-scheme", one can see that the various reaction centers must undergo a precise orchestration of charged and unstable radical species in well defined space-time frames. This requisite is sought when the spatial arrangement is not in a crystal lattice and many crucial components of the scheme are freely mobile and the incident light beam could have diverse intensities, angle of incidence, wavelength spread, etc. The locations of most of the chlorophylls is an outcome of a stochastic distribution. Further, the chlorophylls are not tethered to the lipid layer or the embedded protein via any covalent links 
either (Vernon \& Seely, 1966). How could such stochastically-formed leaf architecture function in reality, with a pre-ordained deterministic Z-scheme mandate for electron flow? This is when the electron needs to go both uphill and downhill (with respect to energy terms), when there are connectivity gaps, when there are both one and two electron exchanges, and that too, up and down the membrane and across macroscopic phases! Via any reasonable rationale, the $\mathrm{Z}$ scheme appears to be an improbable phenomenological scheme. Such a scheme cannot evolve from a minimal set of components and cannot give any productive functioning under a diverse array of environmental conditions, and that, when there is ample oxygen to shunt all such deterministic machineries.

Warburg had originally observed that a given amount of light reduces more $\mathrm{CO}_{2}$ when allowed to fall on the cells intermittently, than continuously. When a frequency of 133 flashes of light were employed per second with equal time periods for light and dark phases, almost a 100\% enhancement in photosynthetic yield could be noted (when compared with the provision of light in a continuous mode). The yield (oxygen produced) was enhanced to $400 \%$ by Emerson, using 50 flashes per second, but with longer dark periods. This means that light was flashed for $3.4 \mathrm{~ms}$ followed by a dark phase of $16.6 \mathrm{~ms}$. Therefore, the slower reaction must be the recharging step of the RC! These data and facts only confirm the analyses made in the earlier paragraphs.

Until now, the electron transfer operations that lead to the final product of ATP/NADPH were discussed. Let us now move onto the fundamental mechanism for oxygen evolution or oxygenesis and study the original data sets of the late 60 s and early 70s, particularly from the pioneering works of Joliot and Kok groups. Since the $\mathrm{Z}$ scheme is rendered redundant, consequentially, the Joliot-Kok cycle of oxygenesis also must be deemed untenable. However, since the oxygenesis observations and inferences thereof led to the deterministic prevailing scheme, they are addressed in detail within the Supplementary Information file. The concerns regarding the Kok-Joliot experimental scheme can be summarized into the following points-

1. Some key observations "taken for granted to be true" were not factually consistent across the work-groups (and sometimes, within the same group too!).

2. Some mathematical assumptions used for the modeling have no theoretical foundations (in structure-function correlation).

3. The experiments were conducted and interpreted with a presumptive and misplaced theoretical basis/bias.

4. Some of the adopted protocols could be questioned, as the analytical tools used fell short of experimental requisites.

5. If we imagine the experimental setup to be an oxygen-limited environment (at the initial state) and also consider that oxygen generation facilitates further catalysis within the system, the initial periodical wavering and the later steady-state values for oxygen can be explained.

Besides the above, one can speculate intuitively and logically that it would be a low probability event that the WSC could hold on to two water molecules and their reaction intermediates for an extended time period, ranging into milliseconds. (Such a paradigm existed in the mOxPhos and $\mathrm{mXM}$ community too, which was recently abrogated with our group's works.) Further, if oxygen were to be involved in electron transfers, then we would expect a consumption of the species within an oxygen limited environment (such as the Kok-Joliot's setups of the first few cycles). 
Therefore, it is imperative that we go beyond the Kok-Joliot model of oxygenesis and explore alternate explanations. Also, high partial pressure of oxygen does not lower rate of oxygen evolution (Haumann et al., 2008). But photosynthesis is lowered at high light intensity and oxygen concentration (the Warburg effect!). This is an outcome that cannot be explained by the prevailing concepts.

\subsection{Interaction of light with pigments and overall energetics:}

Englemann had originally shown with an elegant experiment that the wavelengths of light most efficient for Pl-Pp falls at the two ends of the visible spectrum- "violet" and "red" regions. The action/absorption spectrum shows little correlation with the high quantum yield (photosynthesis efficiency) across the extended region of 400-700 nm (Figure 6). This is observed when the chloroplasts have a preponderence of chlorophyll pigments that absorb/function around the red region. The prevailing "energy" relay systems cannot explain the overall energy capture or harnessing phenomenon, if the electron emission only occurs at PS I and PS II reaction centers, with the unique pigment of Chl $a$ (which absorb "functionally" only at 680-700 nm). The random arrangements of LHCs and CBP leave little scope for any sophisticated ("quantum-physics based logic") mechanism's operation. This is further corroborated by the fact that the current explanation does not account for a higher Chla:Chl $b$ ratio $(2: 1)$ and diverse PS I : PS II chlorophyll varying ratios (1:2 to $3: 2)$. Quite simply, it remains a conundrum as to why should there be so many pigments presented in diverse orientations.

It must be borne in mind that all known matter $\leftrightarrow$ matter and radiation $\leftrightarrow$ matter transfer processes incur loss of energy. Any radiation can interact with matter, and if it alters the state of the matter in any way, it is accompanied with a change in energy term of both, radiation and matter. Further, the efficiency of energy transfers between any two molecules would be critically dependent on distances and orientations. The proposal that a loss-less transfer of energy quantum between homo/hetero species, within a non-crystalline amorphous environment of biomolecular systems "randomly embedded" in lipids and water appears to be a very deterministic proposal indeed. For achieving the transfer of electrons in the quinone and plastocyanic phases, the system is deemed highly fluidic, but for the exciton transfers, the system is deemed to be in solid state. This is like saving the cake for later and savoring it at the same time too!

Thylakoid membranes are amorphous, heterogeneous and anisotropic semi-fluidic structures located in an aqueous pool. The theory of exciton migration was originally developed for relatively crystalline, homogeneous and isotropic assemblies. The evidence for heterogenous energy transfer arises from the ascribing of Chl $a$ fluorescence bands to absorption in wavelengths where Chl $a$ has little absorption (whereas other pigments absorb strongly). It is difficult to envisage homo and hetero energy transfers between various pigments at high efficiencies (with purported values even reaching $100 \%$ ). When the energy transfer efficiency from carotenoids to $\mathrm{Chl} a$ is $10-50 \%$, one cannot explain the action spectrum of photosynthesis. When both the lifetime of excited pigments and fluorescence timeframes fall under the range of nanoseconds, the natural fluorescence would effectively compete with exciton transfer mechanism. Using the observation that a polarized excitation light resulted in depolarized fluorescence as an evidence for homogeneous energy transfer could be erroneous, if DROS were involved as a transient agent. There exists little evidence to support the migration or transfer of electron-hole in the photosynthetic units. 
Forster energy transfer occurs between molecules 2 to $10 \mathrm{~nm}$ apart and for most systems with good orientations, the Forster distance (the spatial separation between the donor and acceptor which yields $50 \%$ energy loss in transmission) is $\sim 5 \mathrm{~nm}$. The multi-protein photosystems' core (not inclusive of the LHCs) measures about $10 \mathrm{~nm}$ or so, and they are separated from each other (homo or hetero distances) by about the same or double that distance or even more. The LHCs dimensions are similar to the photosystem core dimensions but they may be more closely packed with respect to themselves and to the photosystems. The distance between any two photoreceptors (say chlorophyll molecules) is about 6-7 nm, in the least. These findings/arguments lead to a simple statement- energy transmission in such systems cannot be efficient! Recently, a theoretical cum experimental work from Duan et al (2016) has an explicit title- "Nature does not rely on long-lived electronic quantum coherence for photosynthetic energy transfer." Therefore, the mechanism of photon processing and relay remains a highly complex area which few have mastery on, and the field remains "open and yet, closed to all". However, the first electron transfer process from the reaction center chlorophyll to say, a quinone, is fraught with significant loss, but this could render the process significantly downhill. The $\mathrm{Chl}^{*} \rightarrow \mathrm{Chl}^{+}$half reaction has a mid-point potential of $\sim-1000 \mathrm{mV}$ and the two-electron quinone redox couple has a mid-point potential of $\sim-45 \mathrm{mV}$. Using the equation $\boldsymbol{\Delta} \boldsymbol{G}^{\circ}=\mathbf{- n \boldsymbol { J }}$ $\Delta E^{\prime}$, the free energy change of electron transfer between the two couples gives a value of -180 $\mathrm{kJ} / \mathrm{mol}$. Then, one wonders how this ETC can work much greater efficiency, if the overall theoretical efficiency is supposed to be at $39 \%$ !!

It is a daunting experimental task to accurately determine the functional efficiency of the coupling of Pl-Pp and Calvin-Benson cycle. (We have already seen the mentor-mentee difference of opinions in the field, and this would have a clear indication of the difficulties in the area.) This scenario exists because-

(1) It would be practically difficult to account for the (f)actual number of photons of a given wavelength imparted to the leaf/chloroplast from any source of light and the number of quanta absorbed thereafter (within a given amount of time, in a given experimental setup). That is- we know that bright sunlight provides a source of radiation at $\sim 10^{21}$ photons $\mathrm{m}^{-2} \mathrm{~s}^{-1}$. But exactly how many of these photons are absorbed, and how do these absorptions vary with respect to the leaf thickness and position of the cell, etc. are issues that are very difficult to tabulate accurately.

(2) It would be practically difficult to trace the NADPH or ATP molecules formed in vivo or in situ because these products could be dynamically used up. Further, these products' formation route could be switched via cyclic, pseudocyclic and acyclic modalities. Therefore, the actual energy given into the system and the total product output formed by the system cannot be accurately computed experimentally. Therefore, any treatments/arguments of practical values of efficiency shall not be discussed herein.

However, there is a theoretical maximal value that can be set for Pl-Pp, given the prevailing theoretical assumptions. A mole of photons in the visible range (where plant chloroplasts are known to work!) can provide 171 to $299 \mathrm{~kJ}$, corresponding to the wavelengths of 700 to $400 \mathrm{~nm}$. The energy term for synthesizing a mole each of ATP and NADPH is $35 \mathrm{~kJ}$ and $220 \mathrm{~kJ}$ respectively. We know that a single Calvin-Benson cycle scheme requires 3 ATP and 2 NADPH (for the fixation of one molecule of $\mathrm{CO}_{2}$ ). As per the current beliefs, this ratio (3 ATP : 2 $\mathrm{NADPH}$ ) happens to be the precise amount of products formed by the input of 4 electrons (from two different water molecules) into the double $\mathrm{Z}$ scheme (inclusive of Photosystems I and II), 
going through the acyclic modality. Now, let us suppose that PS I (700 nm) and PS II (680 nm) are perfectly synchronized and Einstein's photoelectronic laws apply. Then, 4 photons each of $440871 \mathrm{GHz}(4 \times 176 \mathrm{~kJ} / \mathrm{mol}=704 \mathrm{~kJ} / \mathrm{mol})$ and $428274 \mathrm{GHz}(4 \times 171 \mathrm{~kJ} / \mathrm{mol}=684 \mathrm{~kJ} / \mathrm{mol})$ would be (minimally!) required to power the two photosystems for a 4-electron reaction. That would mean an energy input of $704 \mathrm{~kJ} / \mathrm{mol}$ at PS I and $684 \mathrm{~kJ} / \mathrm{mol}$ at PS II, equaling $\sim 1388$ $\mathrm{kJ} / \mathrm{mol}$. The energy required to make the five "energy-rich" molecules is $\sim 545 \mathrm{~kJ} / \mathrm{mol}$ (ATP $=3$ x $35 \mathrm{~kJ} / \mathrm{mol}=105 \mathrm{~kJ} / \mathrm{mol}$ and $\mathrm{NADPH}=2 \times 220 \mathrm{~kJ} / \mathrm{mol}=440 \mathrm{~kJ} / \mathrm{mol}$; and they add up to give $545 \mathrm{~kJ} / \mathrm{mol})$. That gives a maximal theoretical efficiency of $39 \%(=545 / 1388)$ for the overall PlPp process. This is comparable with the experimentally determined maximal yield of 35-40\% that the most efficient/optimized man-made modern photovoltaic systems afford. In terms of the full photosynthetic cycle (overall coupling of Pl-Pp with Calvin-Benson cycle), since 48 photons are taken (at an average of $173.5 \mathrm{~kJ} / \mathrm{mol}$ ) for the formation of glucose, the efficiency runs down to $\sim 34 \%$. Therefore, the primary loss of energy occurs in the Pl-Pp system (if we assume that 8 photons going through a single $Z$ scheme turnover gives 2 NADPH and 3 ATP molecules).

Exciton interactions are appreciable and measurable only for short distances of $<20 \mathrm{~A}$ for molecules like chlorophyll ( $\sim 5$ times porphyrin's thickness and less than twice the diameter). Whereas Forster resonance interaction may be observed $<100$ A (Kenneth Sauer, Chapter 3 of Bioenergetics book already cited, edited by Govindjee). But then, Forster energy transmission is not efficient! Therefore, the purported scheme of energy transfer (whether via exciton/quantum coherence) from Chl $b \rightarrow$ Lutein $\rightarrow$ Zeaxanthin $\rightarrow \beta$-Carotene $\rightarrow$ Lycopene $\rightarrow$ Chl $a(R C$, P680 or $P 700$ ) does not explain the action spectrum versus absorption spectrum juxtaposition.

Figure 6: A schematic representation of the absorption and photosynthetic efficiency across the visual spectrum of light. The left $Y$-axis is drawn on an arbitrary but relative scale. Experimentally, the minimal/optimal value of $\sim 10$ photons (average of 8 to 12) is needed per oxygen molecule evolution, which is represented as the maximal value on the right $Y$-axis.

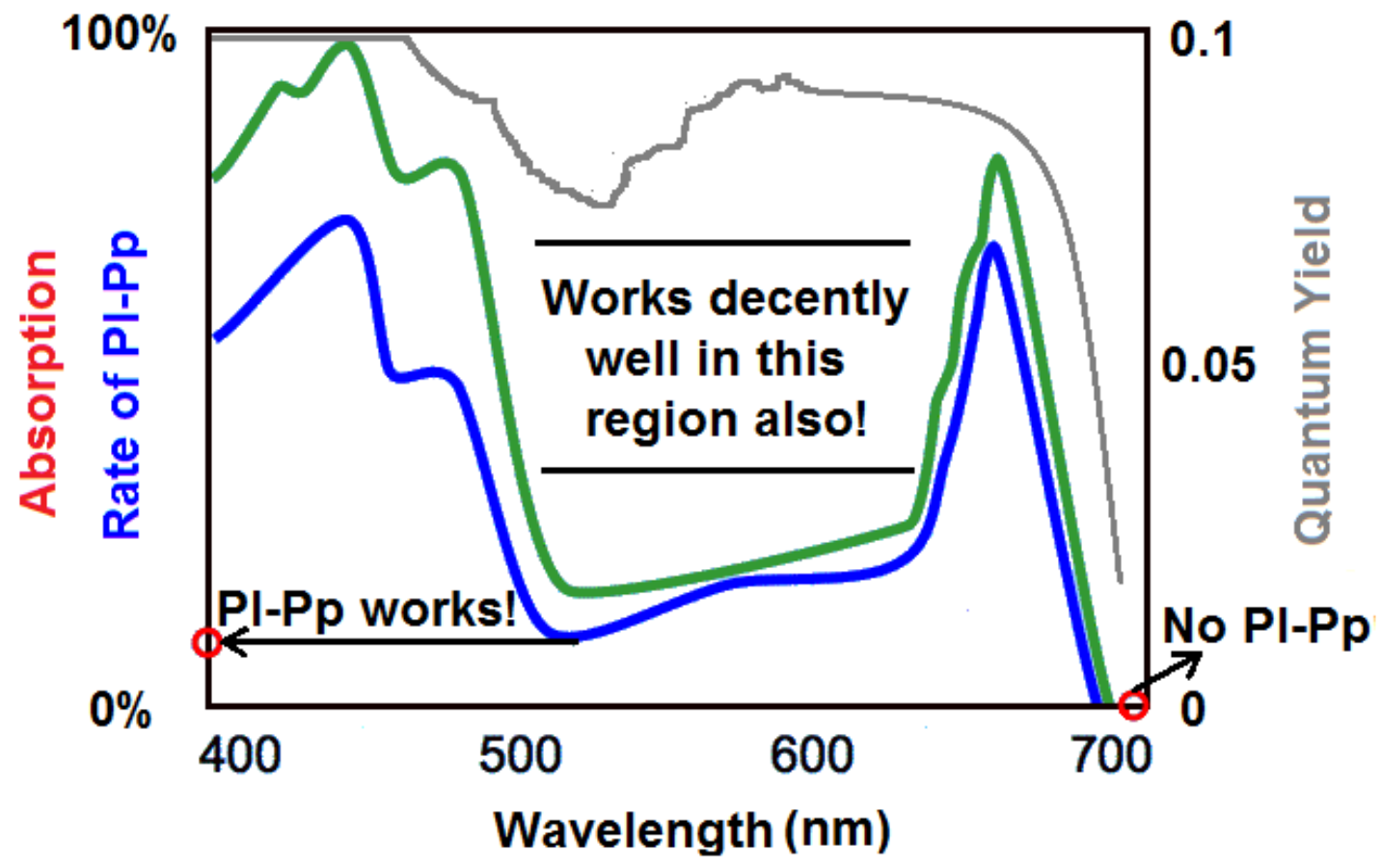




\subsection{The Emerson effect experiment:}

The discovery of the Emerson effect (Emerson et al., 1957; Govindjee et al., 2017) was the turning point in photosynthesis research, and it was the cornerstone that laid the foundation of the modern $\mathrm{Z}$ scheme and Joliot-Kok cycle. A very important fact stands out in the data and this can be seen from several educational websites (quoted herein for the benefit of the current generation students and uninitiated in the field), likehttp://plantphys.info/plant_physiology/light.shtml OR https://biology4isc.weebly.com/c-photosynthesis.html In the first set of illumination, a photosynthetic system of intact PS I cum PS II machinery (say, green algal cells) is exposed to $680 \mathrm{~nm}$ light and $700 \mathrm{~nm}$ light independently. In the respective cases, let's say that about $\mathrm{x}$ and $\mathrm{y}$ amounts of oxygen evolved respectively, where $\mathrm{x} \leq \mathrm{y}$. In the next set, when the same system is exposed to both $680 \mathrm{~nm}$ and $700 \mathrm{~nm}$ light simultaneously, more than $>(x+y)$ oxygen is derived. This observation was taken as the evidence to infer that PS I and PS II are connected in series, and that such a connectivity explains the enhanced yield observed when both photosystems are activated. A cynic would ask- How could oxygen evolve at all in the first set when the system was exposed to only $700 \mathrm{~nm}$ light? This can only fire P700 (or PS I), which cannot break water! This simple long-standing "non-trivial" observation is good enough to discredit the "standing paradigm" of explanation, which shall be henceforth called the ZKJQC (standing for Z-scheme, Kok-Joliot cycle, \& Quantum Coherence/Computing respectively for electron transfer, oxygen evolution and light's interaction with pigments). Since proton pumps, chemiosmosis principle and rotary ATP synthesis have already been discredited, they are not given importance in the acronym.

The next aspect of the Z-scheme is the logic of connectivity. The researchers of later times assumed that for some reason, the series connectivity would better explain the efficiency of electron transfer in the PS I + PS II combine system. This does not make ANY logic from the fundamental perspective of electronic circuitry. Figure 7 shows the analysis of the photosystem machineries in two simple connectivities, series (left) and parallel (right). Though the physiological electron flow is acyclic (electrons derived from water gets stored in NADPH), it would be easily possible to simulate the working logic into the electrical paradigms that we are more familiar. (This can be easily envisaged by imagining a steady-state wherein the NADPH formed is spontaneously broken down for physiological purposes, concomitant with the input of water into the system. Else, the cyclic photosynthesis scheme can also be considered for analogy.) This enables us to analyze the physiological electron flow, with known fundamentals of electronic circuits. Now, PS I and PS II machineries can be deemed as electron-conducting and/or electron-discharging elements. (Induction effects can be safely sidelined as there are no ferromagnetic elements/principles involved.) These functions are the respective electronic principles of conductance (inverse of the ohmic resistance, R) and capacitance (C). If two elements are brought together under the same powering element, enhanced electron flow (current) can be feasible ONLY in the parallel connectivity (and not in series, as Z-scheme is!). Only in the parallel connectivity, the resulting total conductance (1/Tot R) and total capacitance (Tot C) are higher than the two individual components, giving rise to a higher current. So, the series $\mathrm{Z}$ scheme cannot explain the higher yields in the combined illumination setup either!

Figure 7: Analysis of electron flow through two arrangements of PS I and PS II machineries. 

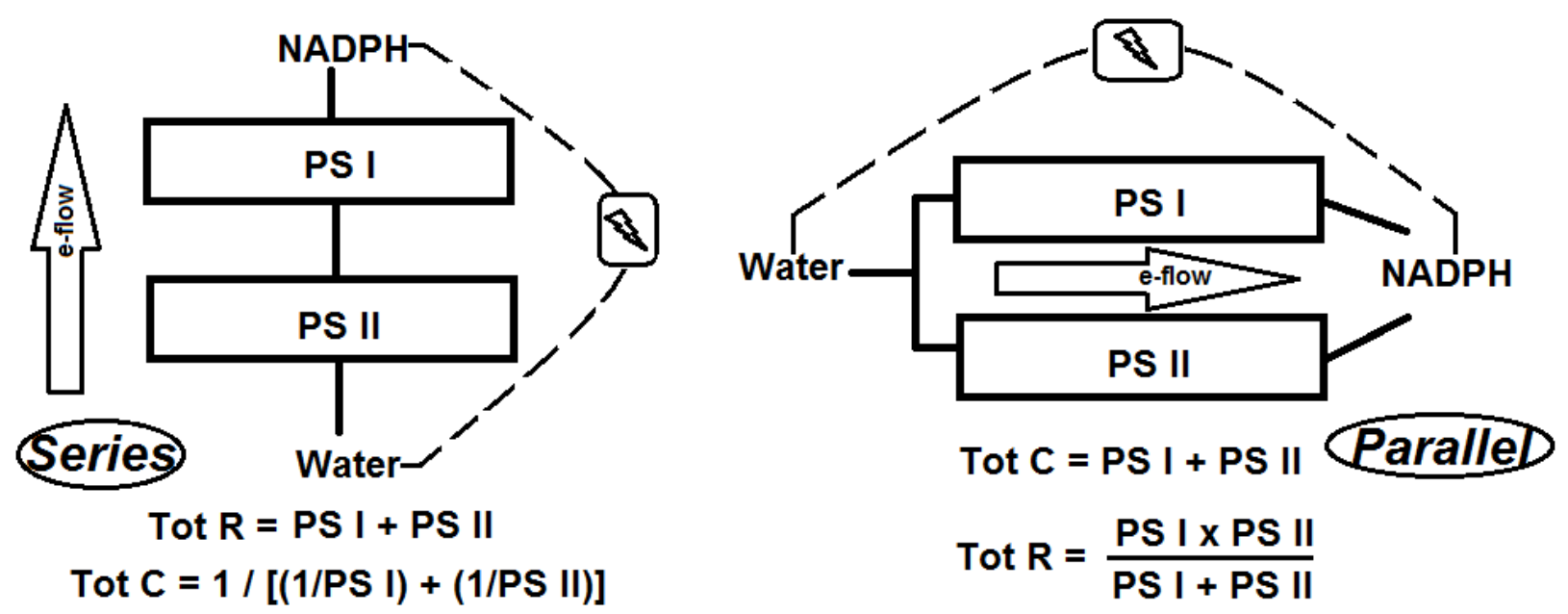

\section{Redirecting principles from related systems}

\subsection{Comparison of mOxPhos and Pl-Pp reaction strategies to draw analogies in the overall phenomenology}

Based on existential analogies between $\mathrm{mXM}$ and mOxPhos, the mechanism of the latter system's mechanistic understanding was revamped. A similar exercise is undertaken herein by comparing mOxPhos with Pl-Pp, as shown in Figure 8. mOxPhos transpires at the inner membrane of mitochondria and is driven majorly by the chemical energy of NADH (produced in Krebs cycle) oxidation. Pl-Pp occurs around the inner membrane of chloroplasts and is driven by radiant energy derived from sunlight. A quick survey of the elements of these two systems would show that mOxPhos and photophos could be deemed similar or anti-/quasi- parallel.

* Both micron-scaled organelle systems employ electron transfer phenomena at their inner phospholipid membrane interface, across distances of $\sim 10^{3} \AA$ per cycle. Also, both processes employ a series of multi-protein complexes with diverse metal centers (hemes, Fe-S proteins, flavins, etc.); and are experimentally shown to liberate heat and ROS during their functioning.

* Both paradigms avail diverse redox systems for supposedly forming intricate Electron Transfer Chains (ETCs), incorporating the common elements of proton pumps, membrane-based organoquinone 2-electron translocator and aqueous-phase based metallo-protein 1-electron translocator. * Overall, it can be seen that both ETC reaction schemes essentially require the input of a total of 4-electrons from two substrate molecules (two NADH/succinate molecules in mOxPhos and two water molecules in Pl-Pp) to give an output of two reduced molecules (two water molecules in mOxPhos and two NADPH molecules in photophos).

* In the ETC of both systems, the first abstraction step purportedly involves the receipt of two/four-electron equivalents from the substrate(s). But before the transfer of the two-electron equivalents to the membrane-harbored quinones in the next step, several one-electron relays are supposedly involved in these highly complicated systems (Complex I and Photosystem II).

Figure 8: A comparison of perceptions on structure-function correlations of mitochondria and chloroplasts, to depict functional parallels in mOxPhos and Pl-Pp systems. The proton-pumps' / 
Complex V's orientations and "electron flow circuitry" and variables in mitochondrial and chloroplast phosphorylation systems are depicted. The contents within the brackets signify the electron acceptance capacity and/or transfer ability of the given component. (Please refer to the list of abbreviations for clarifications, if any needed.) Both systems are also proficient generators of DROS and heat.

Mitochondrion

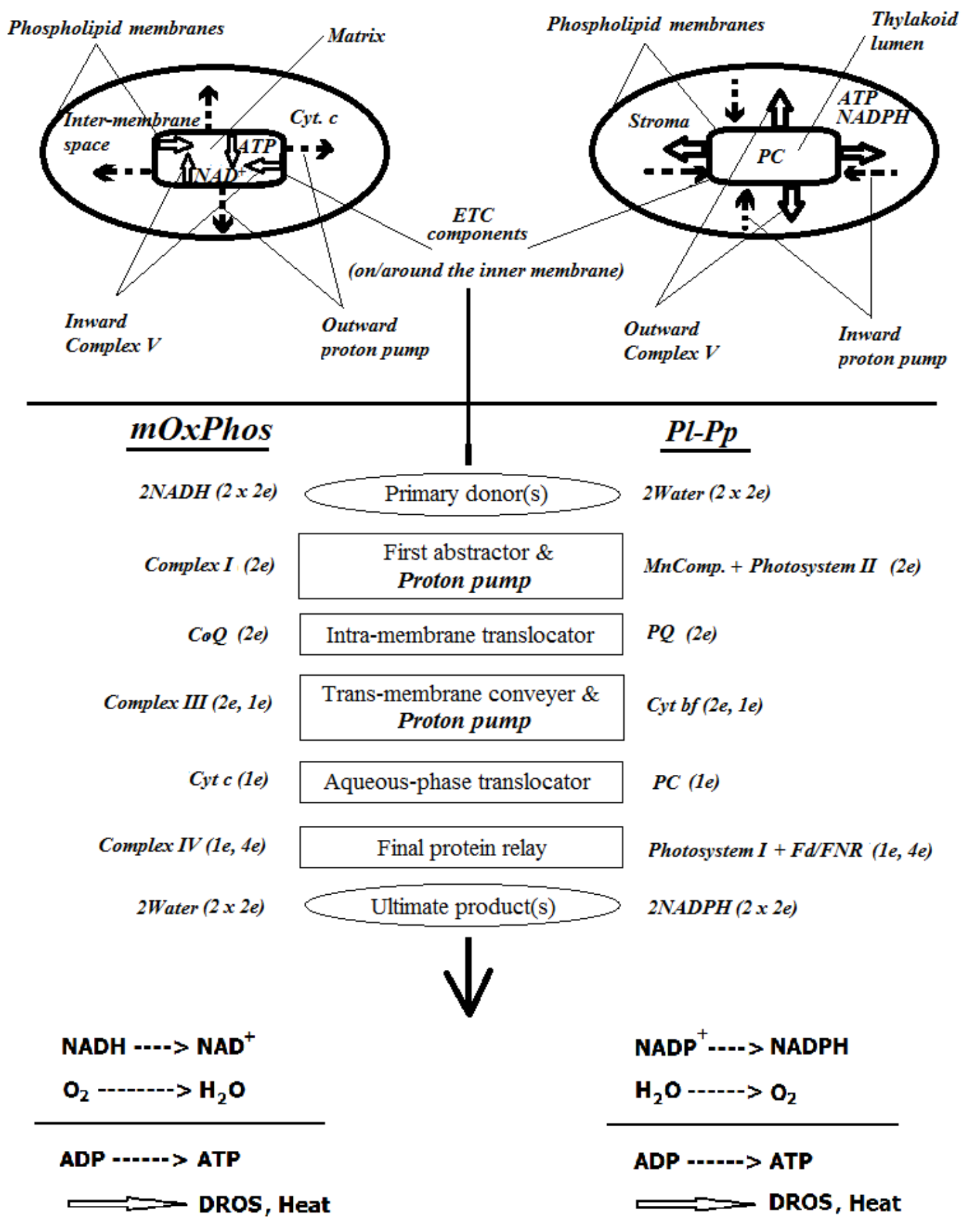

\section{Chloroplast}


* Very strikingly, the element of trans-membrane electron conveyor (called Complex III in mitochondria and Cyt bf in chloroplasts) is astonishingly common, receiving 2-electron equivalents from membrane-quinones and giving away 1-electron equivalents to a disconnected phase.

Therefore, quite like the erstwhile perception of mOxPhos, electrons are supposed to flow through a series of membrane-bound as well as freely-soluble aqueous-phase carriers belonging to various classes like cytochromes, quinones, hemes/chlorophylls, Fe-S proteins; all the while creating a proton gradient across the membrane. Based on conclusive quantitative logic and available experimental data, the prevailing explanation of the "Rotary ATP synthesis Chemiosmosis - Proton pump - Electron Transport Chain (RCPE) were dismantled recently (Manoj, 2017; Manoj, 2018) and an evidence-based murburn scheme was proposed in lieu (Manoj et al. 2019). Though there exist several stark differences between mOxPhos and Pl-Pp (with respect to structure/orientation of catalysts and partitioning of participants), the analogy above shows that several "vitally deterministic and multi-molecular reaction schemes" and the production of DROS/heat are common to both systems. [Also, if the fundamental charging of the Pl-Pp system with photons (zaps $1 \& 2$, the steps where energy is input into the system) is discounted, the rest of the processes are all downhill reaction paradigms that could well fall within the purview of the stochastic murburn scheme.] Therefore, it is natural to infer that the fallacies demonstrated in the RCPE explanations for mOxPhos stand to be relevant for Pl-Pp systems too. Therefore, prima facie / a priori, the mandate is warranted to search for new explanations for Pl-Pp.

\subsection{Cyanobacterial model of OxPhos and Pl-Pp}

Unlike the eukaryotic plants that have both mitochondria and chloroplasts (two distinct organelles to carry out the separate electron flows of OxPhos and Pl-Pp respectively) within their cells, cyanobacteria conduct both photosynthesis (Pl-Pp) and respiration (OxPhos) within their thylakoids. They do so using the common machineries of Cyt $b f$, plastoquinone and $\mathrm{Cyt}_{6}$. In Figure 9, the currently held understanding of cyanobacterial respiratory and photosynthetic metabolism is depicted.

Figure 10A is pictured below to depict the essential requirements of a controllable working model which includes coupled uphill and downhill machineries within a given system. In such a system, the elements of the two facets are distinct and there are separate (inter-connected) reservoirs of the "fuel" synthesized or used. In Figure 10B, the currently prevailing mechanistic impression of the cyanobacterial metabolism is shown. As seen, this model has a propensity to drain (wastefully!), with the given configuration. As soon as the light illumination stops, catabolic activities would drain the redox equivalents, killing the cell. [A battery that is put on a load of " $x$ " watts cannot get simultaneously charged by another capacitor of " $y$ " watts $(y \approx x)$, using the same electro-motive force conduits. An ordinary automobile's battery gets charged and takes up load at the same time owing to a modular setup where the power loop logics are distinctly demarcated. Even in these setups, as soon as charging alternator is taken off, the system would be drained off all power, if the load remains connected.]

Figure 9: A schematic of cell structure and ETC proteins' distribution at the thylakoids of cyanobacteria. 


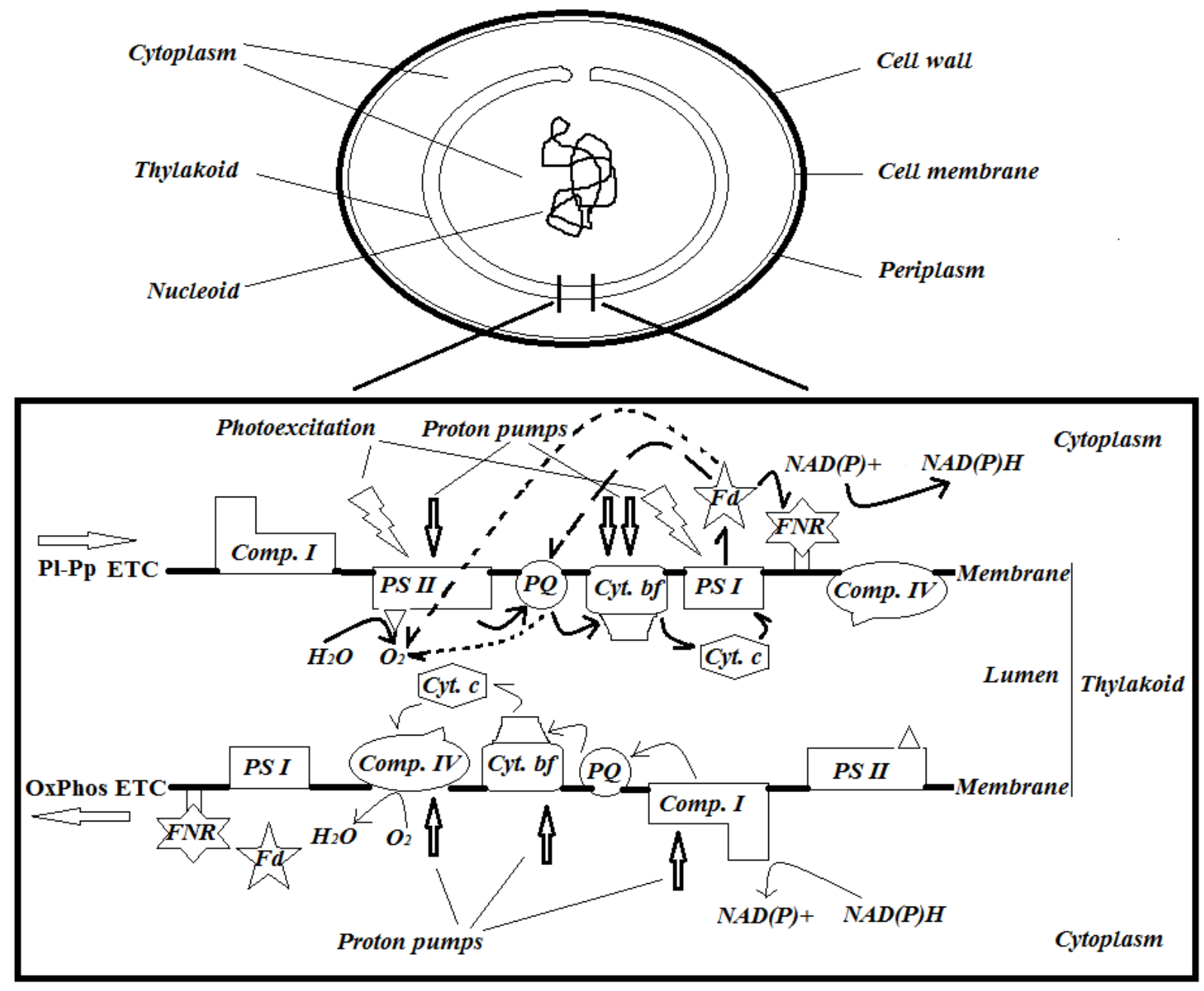

Figure 10: Comparison of controlled working model (A) and spontaneously drained model (B) schemes for cyanobacterial redox metabolism.
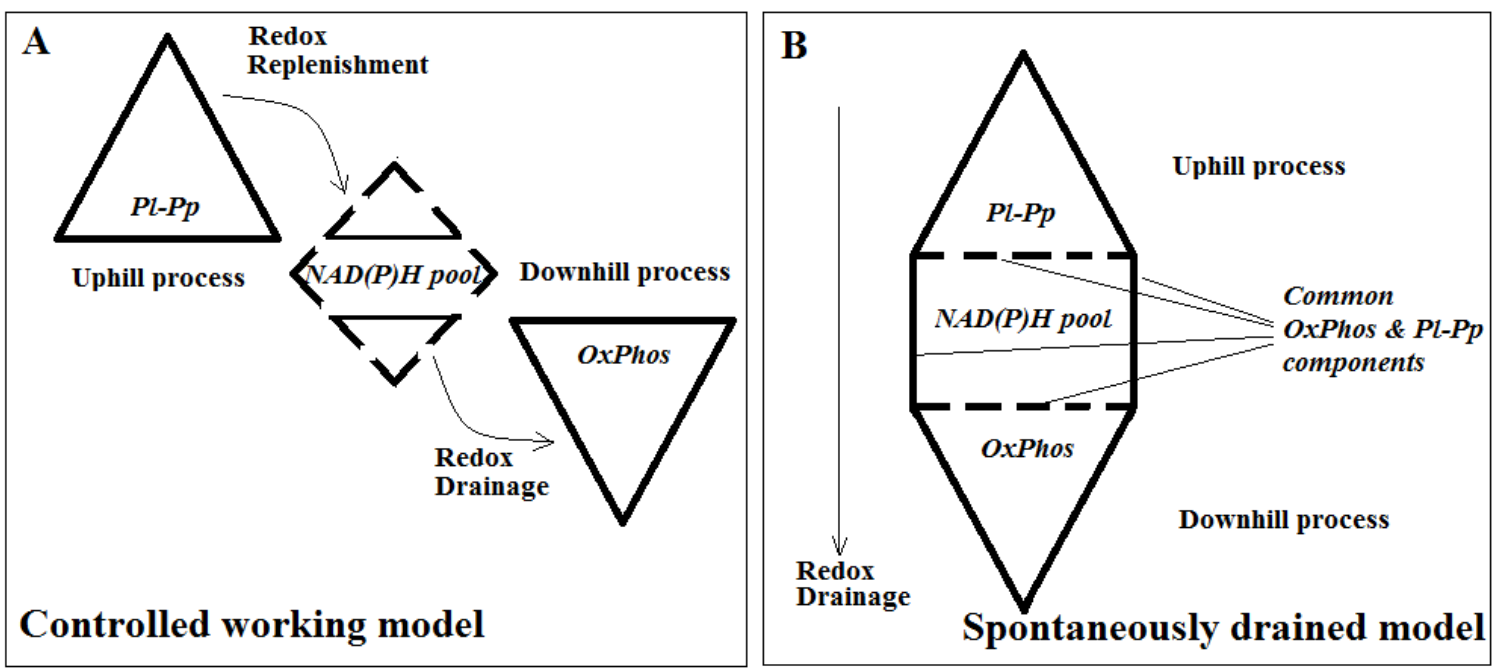
Under the prevailing perspective, it is difficult to see an unintelligent Cyt $c_{6}$ deciding on imparting electrons to PS I or Complex IV. This "decision making" is crucial because it could lead to an "active synthesis" of NADPH or depletion of the same. That is- let us imagine that the bacterium is availing sunlight. Cyt $c_{6}$ would more probably give electrons to Complex IV (rather than a transient electron deficient PS I); and this Complex I would keep breaking down any NADPH that ever formed in the cytoplasm. The resultant would be that photoexcitation would only lead to a thylakoid to cytoplasm "cycling" of protons/water. It is highly unlikely that a protein like Cyt. $c_{6}$ can be instructed to switch between the OxPhos and Pl-Pp cycles, at the demands of the cell. The current mechanism seeks Cyt. $c_{6}$ to "drive up and down the road at the same time", around the membrane within anti-parallel traffics of OxPhos and Pl-Pp. Quite simply, the current mechanistic perspective of ETC is destined to fail!

Now, considering the non-conventional (but proven and accepted!) electron transfer possibilities that several (activated) components of the Pl-Pp system, namely- PS II, PS I, PQ, Fd, NR, etc. (only the last three of these putative interactions are denoted by dashed connectives in Figure 5) could give electron(s) to the omnipresent $\mathrm{O}_{2}$ and DROS, the currently perceived Pl-Pp ETC seems to be just "a wishfully deterministic scheme", and less of a probabilistically relevant pathway. (This statement would be further qualified in the section critiquing ETC below.)

With the best possible orientations and juxtaposition of the proteins and directionalities of proton pumps, there is yet a stark problem with the current understanding. That is- if proton pumps and a deterministic ETC were the key operatives, the very same unintelligent Cyt $b f$ is expected to pump four protons and eight protons respectively into the thylakoid, while indulging in its responsibilities for OxPhos and Pl-Pp cycles (respectively). Why would this mechanistic absurdity result, other than catering to an entrenched traditional mandate? I have already shown that this proton-pumping cannot be managed by the energy term available to Complex III, the mOxPhos equilvalent of Cyt $b f$ (Manoj, 2018). Further, as per the prevailing explanation, there is no justifiable purpose served by having a trans-membrane "electron-routing" step, which would render the "ETC" kinetically non-viable with respect to the overall electron transfer rates. Recent developments in respirasome structures confirm this deduction, rendering the "quinone cycle" a redundant feature of the "forgettable" Mitchellian paradigm. Thus, recent-awareness based critical appraisals severely downplay the hitherto perceived mechanistic understanding.

Very importantly, the smallest photosynthetic cyanobacteria belong to the Prochlorococcus types, which have an average dimension of half a micron. Such a small organism would have practically zilch "free protons" within the cell (let's forget the thylakoids's dimensions!) to serve any proton pumps' needs at physiological $\mathrm{pH}$ ! Therefore, under the clear premise that perceived proton pumps in sub-micron dimensions was just a mirage (and which stands fully debunked!), the current understanding would leave little modalities for ATP and NADPH synthesis. Therefore, a study of the cyanobacterial mOxPhos \& Pl-Pp ETC shows that the hitherto available explanations for electron transfers and driving mechanisms for synthesis are grossly misplaced.

\subsection{Uncoupling proteins of plants and molecules that "uncouple" the $\mathrm{mXM}$, mOxPhos \& Pl- Pp systems}


Uncoupling is a term used in redox enzymology when the enzymes use up the redox equivalents, but do not give fruitful reactions that are physiologically relevant. For example- in the mXM system, NADPH (the source of redox equivalents) could get used up without hydroxylating the xenobiotic substrate. Instead, DROS may get accumulated or water could be formed. Similarly, in the mOxPhos system, NADH gets used forming for heat/DROS formation, without ATP synthesis.

Uncoupling proteins (UCP) in mitochondria cause heat generation and floral thermogenesis is well known in plants. Particularly, the skunk cabbage is famous for its ability to achieve even a $25{ }^{\circ} \mathrm{C}$ higher differential from a near-freezing ambiance (Ito-Inaba, 2014). [A chloroplast-based heat generation process is also known, and this is attribted to non-photochemical quenching (NPQ) at higher light radiance. This is not the topic of discussion here.] The thermogenesis phenomenon was believed to result from a UCP-mediated uncoupling phenomenon, which supposedly led to the dissipation of trans-membrane proton potential (TMP) into heat. Based on new awareness, we have recently shown that UCP-medaited uncoupling and thermogenesis resulted due to its ability to sponsor DROS-based reaction chemistry around the inner mitochondrial membrane (Manoj et al., 2018). This new explanation also provided the rationale for classical uncoupling molecules like dinitrophenol (DNP), which is known to uncouple mOxPhos and Pl-Pp ETCs. [The currently prevailing explanation is unable to explain why UCP enables better photosynthesis (Sweetlove et al. 2006).]

Earlier, in liver microsomes, the N-term peptide sequence of a flavoenzyme, cytochrome P450 reductase (CPR) was supposed to be involved in essential redox partnering with a heme-enzyme, cytochrome P45- (CYP). It was noted that when the N-term segment was cut and left within the reaction medium, significant "uncoupling" resulted. We had shown that the uncoupling mediated by the N-term segment (functionally analogous to UCP/DNP of mitochondria) resulted owing to its ability to modulate DROS (Gideon et al., 2012).

The reaction schemes of $\mathrm{mXM}$ and $\mathrm{mOxPhos}$ are compared with Pl-Pp in Figure 11. It is seen that quite similar to the microsomal and mitochondrial systems, the chlroplast system also leads to uncoupling uncoupling reactions (giving heat and DROS), earlier falsely perceived to be due to "faulty electron transfers" (as summarised in Figure 8). Some molecules like disubstituted phenolics are known to afford uncoupling in $\mathrm{mXM}$, mOxPhos and Pl-Pp systems, implying that the uncoupling mechanism is common across the systems. Since the mXM system has no relevance for proton-pumps and since the proton-potential based explanation for mOxPhos system has been conclusively shown to be untenable, it is imperative that the erstwhile protonpotential based explanation for Pl-Pp is questioned and the feasibility of DROS-based murburn scheme be explored to explain the affects/effets observed. This is because all three systems are simple distributions of protein complexes on lipid membranes and Occam's razor suggests that a simple theory is more plausible than a highly twisted deterministic theory.

Figure 11: The similarities between the productive reactions and uncoupling phenomena observed in mXM, mOxPhos and Pl-Pp systems. Uncoupled reaction outcome is given in the box on the right. 


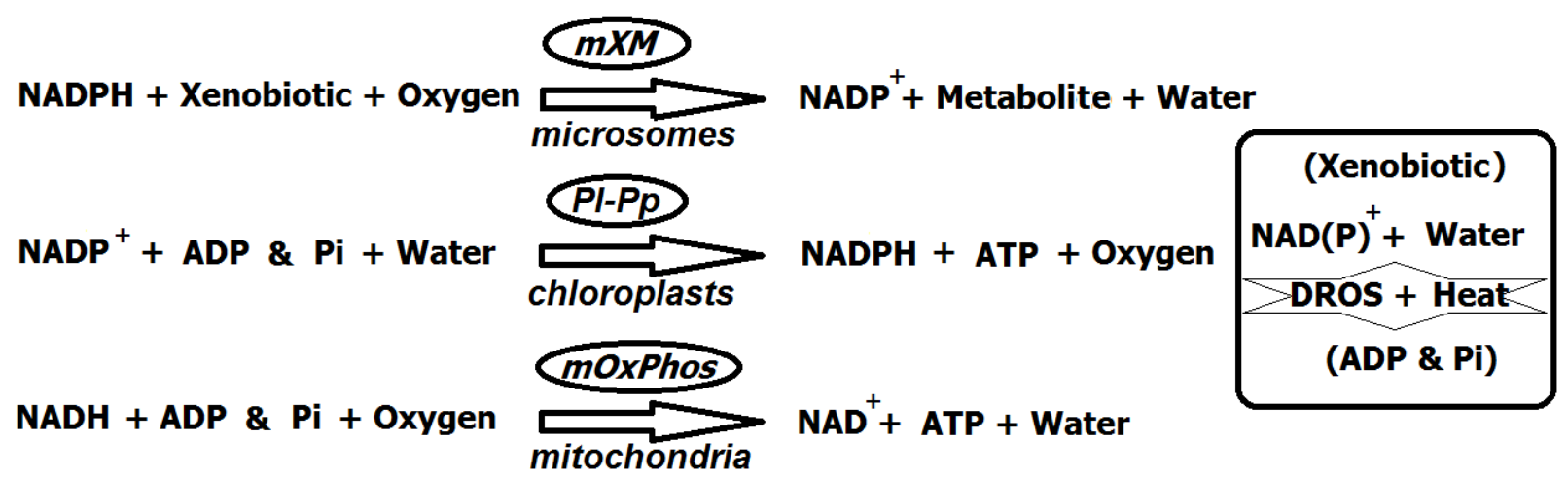

For deriving mechanistic clues of any reaction system, one of the easiest probes is to introduce molecules with known functions and correlate it with observed outcomes.

* If Pl-Pp ETC only employs Marcus' outer sphere model of electron transfer, how can several man-made dyes and redox active molecules put in and draw out electrons from the purportedly deterministic ETC (Hauska, 1977)?

* The prevailing explanations cannot rationalize the unusual concentration-dependent effects brought about by certain additives in Pl-Pp systems (Avron \& Shavit, 1965; Watling-Payne \& Selwyn, 1974). These are typical manifestations of the operational relevance of murburn scheme (Parashar et al., 2018).

* With the Pl-Pp ETC, it is inexplicable as to why cyanide could serve as a toxic principle to the Pl-Pp process (Berg \& Krogmann 1975). Photosystem I (whose functioning is supposedly inhibited by cyanide) is not known to have any Fe-hemes and the inhibition is not owing to any binding-based effects either. Emerson showed that the dark process is temperature sensitive but the light process is not (with respect to oxygen yield). At low temperature, $10 \mathrm{uM}$ cyanide gave higher inhibition at short darkness duration or continuous light, whereas it gave no inhibitory effect at longer dark periods. Such outcomes can be reasoned out within the murburn scheme owing to cyanide's ability to modulate DROS (Parashar et al., 2014; Manoj et al., 2016; Manoj et al., 2018)

* The fact that cations affect the energy transfers within the system (as exemplified by the works of Prasanna Mohanty in Govindjee's group in the early 1970s) is a support of murburn concept.

Given the commonality of reaction schemes and the effects of additive generated outcomes, a simple chemical reaction scheme is more probable and murburn concept fits the bill.

\subsection{Bacterial rhodopsin and the "chemical trapping” of light energy}

Rhodopsin is a rather simple protein (a monomer) with seven trans-membrane helices. The pigment or cofactor in rhodopsin is a simple retinoid molecule, called retinal. The current mechanism for retinal-based photo-induced processes involve cis-trans isomerizations (and associated conformational change in the protein), leading to specific amino acid residues' protonations and deprotonations. These processes are supposed to lead to a proton pumping 
effect. Several research groups (working on different rhodopsins in different time periods) have found that at physiologically relevant regimes (at slightly above neutral $\mathrm{pH}$, with moderate ionic strength), the $\mathrm{pK}_{\mathrm{a}}$ of lysine conjugated retinal Schiff's base ranges from a value of 10 to 16, with most groups placing it to 13 (Druckmann et al., 1982; Sheves et al., 1986; Balashov et al., 1991; Steinberg et al., 1993; Liang et al. 1994; Govindjee et al. 1994; Rousso et al., 1995)! It is highly unlikely that this species would impart a proton to the side-chain of an aspartate residue (as the currently prevailing mechanistic interpretation solicits!) or any other species at physiological $\mathrm{pH}$ ranges. With the available understanding of chemico-physics, it is highly unlikely that such a protein-based mechanism could serve as trans-membrane proton pumps.

Therefore, when seeing that (under sub-micro regimes)- (i) there are no source of free protons, (ii) there are little ways that a proton gradient can be efficiently tapped by a biological system, and (iii) the mOxPhos proton pump model has been conclusively debunked- it would make a lot of chemical sense to seek an alternate explanation for the mechanism of rhodopsin functioning. It appears more likely that the covalent modification with lysine is enabled to tether the retinal into the protein within the trans-membrane region (to enable murcat process) and enhance the electron density even fruther by conjugating the sulphur lone pairs into the system.

Now, an alternate hypothesis can also be entertained. Retinal is a pi-conjugated system, known well to get excited by light, and also known to generate reactive species by a diverse array of envisaged/probable mechanisms (Aboltin et al 2013). Retinal can be excited by light to give its excited form. The essential process is represented by-

$\mathrm{R}+\mathrm{h} v \rightarrow \mathrm{R}^{*}$

The excited or native species can lose/gain an electron and/or thus lead to several transiently stabilized species within the system.

$\mathrm{R}^{*} \rightarrow \mathrm{R}+\mathrm{e}$

$\mathrm{R}$ or $\mathrm{R}^{*}+\mathrm{e} \rightarrow \mathrm{R}^{\cdot-}$

Else, it can also crossover to triplet state and react with oxygen.

$\mathrm{R}^{*} \rightarrow{ }^{3} \mathrm{R}^{*}$

${ }^{3} \mathrm{R} *+{ }^{3} \mathrm{O}_{2} \rightarrow \mathrm{R}+{ }^{1} \mathrm{O}_{2}$

Now, in such an oxygenic system, superoxide could be formed in a multitude of ways.

$\mathrm{O}_{2}+\mathrm{e} \rightarrow \mathrm{O}_{2}^{--}$

$\mathrm{R}^{*}+\mathrm{O}_{2} \rightarrow \mathrm{R}^{\cdot+}+\mathrm{O}_{2}^{\cdot-}$

$\mathrm{R}^{\cdot-}+\mathrm{O}_{2} \rightarrow \mathrm{R}+\mathrm{O}_{2}^{\cdot-}$

Thereafter, within a partially closed and heterogenous system (with specific transport/partioning principles), directional electron transfer could spontaneously set in place, yielding continuous source of superoxide species. Dismutation of superoxide $\left(\mathrm{pK}_{\mathrm{a}}\right.$ is on the acidic scale) gives peroxide $\left(\mathrm{pK}_{\mathrm{a}}\right.$ is on the alkaline scale) and the Haber-Weiss chemistry that results can result in hydroxyl radicals. These transitions and reactions lead to "breaking" of water and release of protons in the milieu. The site of proton release would depend on the orientation of the retinal moiety within the trans-membrane region of the rhodopsin molecule (and the accompanying relay networks of the amino acid residues that ensconse the retinal molecule) and the extent of 
ROS detection in the milieu would be dependent on the efficiency of the quenching or cycling systems around. This phenomenon could also be inadvertently or indirectly referred to as a "proton pump". These DROS based mechanisms also gain significance from an initial state considerations. (The small organelles lack adequate amounts of protons for the exceeding amounts of proteins to work! This aspect is discussed in detail below.) Therefore, the simple chemical logic of explaining rhodopsin's role in sponsoring ATPsynthase activity also accounts for Racker et al.'s experiment of the past (Racker \& Stoeckenius, 1974) and those like Otrin et al's experiment (Otrin et al., 2017) in recent times. Clearly, the common element in all these systems is DROS (murburn concept), and not proton pumps!

\section{Conclusion}

The theoretical premises that led to the "establishment" of Z-scheme and Kok-Joliot cycle have been successfully critiqued in the current write-up. Both grana and stroma fractions contain PC and the number of plastocyanin is relatively lower, with respect to the expected values for an effective Z-scheme conduction. Though the $\mathrm{Cu}$-ligation sphere is conserved, the topography/surface electrostatics of PC is diverse across the plant kingdom. Such data are strongly against the attributed role of PC serving as a specific translocating conduit between cytochrome $b f$ and PS I. To explain the PS I - PS II interactions, a new mechanistic model is warranted, which connects the components in a parallel modality. Murburn concept can potentially afford such a scheme. The fact that electrons can be put in and withdrawn via several molecules (Hill reagent, for example), the presence of several shunts (Figure 9), various ions and molecules can enhance or affect electron transfers in a maverick concentration-dependent manner, etc. are typical evidence for the relevance of murburn concept in Pl-Pp. Also, the structures of PS I and PS II (position of diverse pigments) may vary within the same plant species and amongst species, based on environment. This signifies that there is no inherent coded information within the various proteins for their positioning the pigments. Also, it would imply that there are no specific circuitries and the chlorophylls and pigments acquire random positions, as permitted during assembly. Further, the half-lives of these photosystems vary by almost two orders, and therefore, the series connectivity between the two photosystems is just non-viable! Chlorophylls are non-covalently bound to protein. Therefore, there cannot be a destined path for photon, energy or electron transfer. There is no evidence to support the notion that chlorophyll is present in the membranes under a crystalline environment. Neither the absorption nor fluorescence is strongly polarized, indicating that the pigments are not ordered in any simple periodic lattices. While the fluorescence yield is high for chlorophyll in solution, it gives almost an order lesser yield in situ. For a molecule like chlorophyll, exciton interactions are appreciable and measurable only for distances less than $2 \mathrm{~nm}$ (a few folds of its linear dimensions). These arguments suggest that diverse chlorophyll molecules are subject to different microenvironments. In such scenarios, it is inappropriate to extrapolate the results found with ordered pigments' photosynthetic structures found in cyanobacteria to that of the random array in plants (Engel at al., 2007). In this context, one must reflect the opinion of (Duan et al, 2017), "The conditions under which matter displays quantum features and biological functionality are contrarious." Therefore, we should look for simpler models to explain photosynthesis, and it would be unwise to suppose that plants are capable of quantum computing (Biello, 2007)! These premises call for the application of a stochastic mechanistic scheme, like murburn concept, which shall be the focus of a forthcoming write-up. At this juncture, it is opportune to end with a 
question- Since there is hardly any difference between Chla at the RC (other than the dimeric status) and Chla elsewhere, why should only the RC Chl $a$ undergo ET upon photoexcitation?

Disclaimer: There are no conflicts of interests involved.

Acknowledgment: The work was powered by Satyamjayatu: The Science \& Ethics Foundation.

\section{REFERENCES}

Aboltin P V, Shevchenko TF, Shumaev KB, Kalamkarov GR. [Photoinduced production of reactive oxygen species by retinal derivatives and conjugates]. Biofizika.

2013;58(2):246-251.

Ahrens W. Herbicide Handbook, seventh edition. Weed Science Society of America, Champaign, IL, 1994.

Anderson WP. Weed Science - Principles and Applications, third edition. West Publishing, NY, 1996.

Andrew D, Hager L, Manoj KM. The intriguing enhancement of chloroperoxidase mediated one-electron oxidations by azide, a known active-site ligand. Biochem Biophys Res Commun. 2011;415(4):646-649.

Avron M, Shavit N. Inhibitors and uncouplers of photophosphorylation. Biochim Biophys Acta - Biophys Incl Photosynth. 1965;109(2):317-331.

Balashov SP, Govindjee R, Ebrey TG. Redshift of the purple membrane absorption band and the deprotonation of tyrosine residues at high $\mathrm{pH}$ : Origin of the parallel photocycles of trans-bacteriorhodopsin. Biophys J. 1991;60(2):475-490.

Bay Z, Pearlstein RM. A theory of energy transfer in the photosynthetic unit. Proc Natl Acad Sci U S A. 1963;50(6):1071-1078.

Benson SL, Maheswaran P, Ware MA, et al. An intact light harvesting complex I antenna system is required for complete state transitions in Arabidopsis. Nat Plants. 2015;1:15176.

Berg SP, Krogmann DW. Mechanism of KCN inhibition of photosystem I. J Biol Chem. 1975;250(23):8957-8962.

Berg JM, Tymoczko JL, Stryer L. Biochemistry. 5th. WH Freeman, New York, 2002.

Biello D. When It Comes to Photosynthesis, Plants Perform Quantum Computation. Scientific American April 13 (2007). 
Blackwell M, Gibas C, Gygax S, Roman D, Wagner B. The plastoquinone diffusion coefficient in chloroplasts and its mechanistic implications. Biochim Biophys Acta Bioenerg. 1994;1183(3):533-543.

Brand J, Baszynski T, Crane FL, Krogmann DW. Photosystem I inhibition by polycations. Biochem Biophys Res Commun. 1971;45(2):538-543.

Caffarri S, Tibiletti T, Jennings RC, Santabarbara S. A comparison between plant photosystem I and photosystem II architecture and functioning. Curr Protein Pept Sci. 2014;15(4):296-331.

Druckmann S, Ottolenghi M, Pande A, Pande J, Callender RH. Acid-base equilibrium of the Schiff base in bacteriorhodopsin. Biochemistry. 1982;21(20):4953-4959.

Devine MD, SO Duke, and C Fedtke. Physiology of Herbicide Action. Prentice Hall, NJ, 1993.

Duan H-G, Prokhorenko VI, Cogdell RJ, et al. Nature does not rely on long-lived electronic quantum coherence for photosynthetic energy transfer. Proc Natl Acad Sci U S A. 2017;114(32):8493-8498.

Emerson R, Chalmers RV, Cederstrand CN. Some factors influencing the long wave limit of photosynthesis. Proc Natl Acad Sci U S A.1957; 43:133-143.

Engel GS, Calhoun TR, Read EL, et al. Evidence for wavelike energy transfer through quantum coherence in photosynthetic systems. Nature. 2007;446(7137):782-786.

Franck J, Teller E. Migration and Photochemical Action of Excitation Energy in Crystals. J Chem Phys. 1938;6(12):861-872.

Fromme P, Jordan P, Krauss N. Structure of photosystem I. Biochim Biophys Acta. 2001;1507(1-3):5-31.

Gade SK, Bhattacharya S, Manoj KM. Redox active molecules cytochrome c and vitamin $\mathrm{C}$ enhance heme-enzyme peroxidations by serving as non-specific agents for redox relay. Biochem Biophys Res Commun. 2012;419(2):211-214.

Gideon DA, Kumari R, Lynn AM, Manoj KM. What is the Functional Role of N-terminal Transmembrane Helices in the Metabolism Mediated by Liver Microsomal Cytochrome P450 and its Reductase? Cell Biochem Biophys. 2012;63(1):35-45.

Govindjee. On the requirement of minimum number of four versus eight quanta of light for the evolution of one molecule of oxygen in photosynthesis: A historical note.

Photosynth Res. 1999;59(2):249-254. 
Govindjee R, Balashov S, Ebrey T, Oesterhelt D, Steinberg G, Sheves M. Lowering the intrinsic $\mathrm{pKa}$ of the chromophore's Schiff base can restore its light-induced deprotonation in the inactive Tyr-57-->Asn mutant of bacteriorhodopsin. J Biol Chem.

1994;269(20):14353-14354.

Govindjee, Shevela D, Bjorn LO. Evolution of the Z-scheme of photosynthesis: a perspective. Photosynth Res. 2017;133(1-3):5-15.

Govindjee. Bioenergetics of photosynthesis. Academic Press, New York, 1975.

Haehnel W, Ratajczak R, Robenek H. Lateral distribution and diffusion of plastocyanin in chloroplast thylakoids. J Cell Biol. 1989;108(4):1397-1405.

Haumann M, Grundmeier A, Zaharieva I, Dau H. Photosynthetic water oxidation at elevated dioxygen partial pressure monitored by time-resolved X-ray absorption measurements. Proc Natl Acad Sci U S A. 2008;105(45):17384-17389.

Hauska, G. (1977). "Artificial acceptors and donors," in Encyclopedia of Plant Physiology, eds. A. Trebst \& M. Avron. (Berlin: Springer Berlin Heidelberg), 253-265.

Ito-Inaba Y. Thermogenesis in skunk cabbage (Symplocarpus renifolius): New insights from the ultrastructure and gene expression profiles. Adv Hortic Sci Vol 28, No 2. June 2014.

Jagendorf AT, Uribe E. ATP formation caused by acid-base transition of spinach chloroplasts. Proc Natl Acad Sci U S A. 1966;55(1):170-177.

Kirchhoff H. Diffusion of molecules and macromolecules in thylakoid membranes. Biochim Biophys Acta. 2014;1837(4):495-502.

Lehninger AL, Nelson DL, Cox M. Principles of Biochemistry: Palgrave Macmillan Limited, 2004.

Leo P. Vernon, Gilbert R. Seely. The Chlorophylls. 1st Edition Academic Press, 1966.

Liang J, Steinberg G, Livnah N, Sheves M, Ebrey TG, Tsuda M. The pKa of the protonated Schiff bases of gecko cone and octopus visual pigments. Biophys J. 1994;67(2):848-854.

Manoj KM, Venkatachalam A, Parashar A. Metabolism of xenobiotics by cytochrome P450: novel insights into the thermodynamics, kinetics and roles of redox proteins and diffusible reactive species. In: Drug Metabolism Reviews. ; 2016:41-42.

Manoj KM. Debunking Chemiosmosis and Proposing Murburn Concept as the Operative Principle for Cellular Respiration. Biomed Rev. 2018;28:31-48. 
Manoj KM, Baburaj A, Ephraim B, et al. Explaining the atypical reaction profiles of heme enzymes with a novel mechanistic hypothesis and kinetic treatment. PLoS One.

2010;5(5):e10601.

Manoj KM, Gade SK, Venkatachalam A, Gideon DA. Electron transfer amongst flavoand hemo-proteins: diffusible species effect the relay processes, not protein-protein binding. RSC Adv. 2016;6(29):24121-24129.

Manoj KM, Gade SK, Mathew L. Cytochrome P450 reductase: a harbinger of diffusible reduced oxygen species. PLoS One. 2010;5(10):e13272.

Manoj KM, Hager LP. Chloroperoxidase, a Janus enzyme. Biochemistry. 2008;47(9):2997-3003.

Manoj KM, Parashar A, Gade SK, Venkatachalam A. Functioning of Microsomal Cytochrome P450s: Murburn Concept Explains the Metabolism of Xenobiotics in Hepatocytes. Front Pharmacol. 2016;7:161.

Manoj KM, Parashar A, Venkatachalam A, et al. Atypical profiles and modulations of heme-enzymes catalyzed outcomes by low amounts of diverse additives suggest diffusible radicals' obligatory involvement in such redox reactions. Biochimie. 2016;125:91-111.

Manoj KM. Chlorinations catalyzed by chloroperoxidase occur via diffusible intermediate(s) and the reaction components play multiple roles in the overall process. Biochim Biophys Acta. 2006;1764(8):1325-1339.

Manoj KM. Aerobic respiration: Criticism of the proton-centric explanation of rotary ATP synthesis, chemiosmosis principle, proton pumps and electron transport chain. In Press (2018).

Manoj KM, Parashar A, Jacob VD, Ramasamy S. Aerobic respiration: Proof of concept for the murburn perspective. In Press (2018).

Manoj KM, Gideon DA, Jacob VD, Ramasamy S. Murburn explanation for thermogenesis mediated by uncoupling protein. In Press. (2018).

Mazor Y, Borovikova A, Caspy I, Nelson N. Structure of the plant photosystem I supercomplex at 2.6 ̊̊ resolution. Nat Plants. 2017;3:17014.

Mitchell P. Coupling of phosphorylation to electron and hydrogen transfer by a chemiosmotic type of mechanism. Nature. 1961;191:144-148.

Nelson N, Ben-Shem A. The complex architecture of oxygenic photosynthesis. Nat Rev Mol Cell Biol. 2004;5(12):971-982. 
Nelson N, Yocum CF. Structure and function of photosystems I and II. Annu Rev Plant Biol. 2006;57:521-565.

Nicholls DG. Mitochondrial membrane potential and aging. Aging Cell. 2004;3(1):35-40.

Otrin L, Marusic N, Bednarz C, et al. Toward Artificial Mitochondrion: Mimicking Oxidative Phosphorylation in Polymer and Hybrid Membranes. Nano Lett.

2017;17(11):6816-6821.

Panitchayangkoon G, Voronine D V, Abramavicius D, et al. Direct evidence of quantum transport in photosynthetic light-harvesting complexes. Proc Natl Acad Sci U S A. 2011;108(52):20908-20912.

Parashar A, Gade SK, Potnuru M, Madhavan N, Manoj KM. The curious case of benzbromarone: insight into super-inhibition of cytochrome P450. PLoS One. 2014;9(3):e89967.

Parashar A, Gideon DA, Manoj KM. Murburn Concept: A Molecular Explanation for Hormetic and Idiosyncratic Dose Responses. Dose Response. 2018;16(2):1559325818774421.

Parashar A, Manoj KM. Traces of certain drug molecules can enhance heme-enzyme catalytic outcomes. Biochem Biophys Res Commun. 2012;417(3):1041-1045.

Parashar A, Venkatachalam A, Gideon DA, Manoj KM. Cyanide does more to inhibit heme enzymes, than merely serving as an active-site ligand. Biochem Biophys Res Commun. 2014;455(3-4):190-193.

Philipson KD, Sauer K. Exciton interaction in a bacteriochlorophyll--protein from Chloropseudomonas ethylica. Absorption and circular dichroism at 77 degrees $\mathrm{K}$. Biochemistry. 1972;11(10):1880-1885.

Racker E, Stoeckenius W. Reconstitution of purple membrane vesicles catalyzing lightdriven proton uptake and adenosine triphosphate formation. $J$ Biol Chem.

1974;249(2):662-663.

Ross MA and CA Lembi. Applied Weed Science, second edition. Prentice Hall, NJ, 1999.

Rousso I, Friedman N, Sheves M, Ottolenghi M. pKa of the protonated Schiff base and aspartic 85 in the bacteriorhodopsin binding site is controlled by a specific geometry between the two residues. Biochemistry. 1995;34(37):12059-12065.

Sanderson DG, Anderson LB, Gross EL. Determination of the redox potential and diffusion coefficient of the protein plastocyanin using optically transparent filar electrodes. Biochim Biophys Acta. 1986;852(2-3):269-278. 
Sheves M, Albeck A, Friedman N, Ottolenghi M. Controlling the pKa of the bacteriorhodopsin Schiff base by use of artificial retinal analogues. Proc Natl Acad Sci U S A. 1986;83(10):3262-3266.

Steinberg G, Ottolenghi M, Sheves M. pKa of the protonated Schiff base of bovine rhodopsin. A study with artificial pigments. Biophys J. 1993;64(5):1499-1502.

Sweetlove LJ, Lytovchenko A, Morgan M, et al. Mitochondrial uncoupling protein is required for efficient photosynthesis. Proc Natl Acad Sci U S A. 2006;103(51):1958719592.

Venkatachalam A, Parashar A, Manoj KM. Functioning of drug-metabolizing microsomal cytochrome P450s: In silico probing of proteins suggests that the distal heme "active site" pocket plays a relatively "passive role" in some enzyme-substrate interactions. silico Pharmacol. 2016;4(1):2.

Voet D and Voet JG. Biochemistry. 4th: Wiley, Hoboken, NJ, USA, 2011.

Watling-Payne AS, Selwyn MJ. Inhibition and uncoupling of photophosphorylation in isolated chloroplasts by organotin, organomercury and diphenyleneiodonium compounds. Biochem J. 1974;142(1):65-74.

Wei X, Su X, Cao P, et al. Structure of spinach photosystem II-LHCII supercomplex at 3.2 A resolution. Nature. 2016;534(7605):69-74.

Young ID, Ibrahim M, Chatterjee R, et al. Structure of photosystem II and substrate binding at room temperature. Nature. 2016;540(7633):453-457. 


\title{
Supplementary Information File
}

\section{Oxygenic photosynthesis: Critiquing the standing explanations and proposing explorative solutions based in murburn concept}

\author{
Kelath Murali Manoj* \\ * Satyamjayatu: The Science \& Ethics Foundation \\ Kulappully, Shoranur-2 (PO), Palakkad District, Kerala State, India-679122. \\ Email: satyamjayatu@yahoo.com
}

\section{$\underline{\text { An analysis of the Kok-Joliot cycle from pioneers' data }}$}

The observation that dark-adapted chloroplasts fail to produce oxygen with a single flash (Allen and Franck, 1955) inspired the direction of research in photosynthesis, thereafter. Subsequently, Joliot's and Kok's groups had explored and established new techniques/analyses in the field. They interpreted that the initial periodicity of oxygen evolution shown by dark-adapted chloroplasts to a series of short flashes was owing to a "cooperation of charges among labile intermediates within chloroplasts". Essentially, the works implied that oxygen evolution occurs at one site alone, and that after a series of four incremental steps. Herein, I shall re-analyze and re-present the data to investigate if the assumptions and scheme advocated by Joliot/Kok are valid. (I would like to disclaim that a critical analyses of the pioneers' meticulous works does not connote disrespect at my end. I am just trying to search for more meaningful explanations here.)

The left panel of Figure SI1 recaptures the pioneers' data that went on to define the oxygenesis paradigm prevailing today. [The discussion in this section uses the content of the chapter titled "Oxygen evolution in photosynthesis", authored by Pierre Joliot and Bessel Kok, from the book "Bionenergetics", edited by Govindjee (1975). The respective figures were printed out, digitalized and re-plotted/re-analyzed.] The essential observations founding this paradigm are (and I quote verbatim from Crofts' website, UIUC)-

1. No $\mathrm{O}_{2}$-evolution on the first flash, very little $\left(0.2 X Y_{S S}\right)$ after the second, a maximal yield $\left(2.5 X Y_{S S}\right)$ after the third, and a substantial yield (1.3X $\left.Y_{S S}\right)$ after the fourth (where $Y_{S S}$ is

the flash yield in the steady-state). In similar experiments using shorter laser flashes (20 $n s)$ it has been shown that no $\mathrm{O}_{2}$ is evolved on the second flash.

2. A maximum in $\mathrm{O}_{2}$-evolution every fourth flash after the third.

3. A damping of the amplitude of the oscillation.

Figure SI1: The oxygen evolution pattern reported by Joliot and Kok research groups (when darkacclimatized chloroplasts, pre-primed with suitable light flash) were exposed to periodic light flashes. On the right, simulations are made wherein the initial state is perpetuated as such (sans approach to the "steady state" that is experimentally observed), without double hits and/or misses. 
Pioneers' experimental data

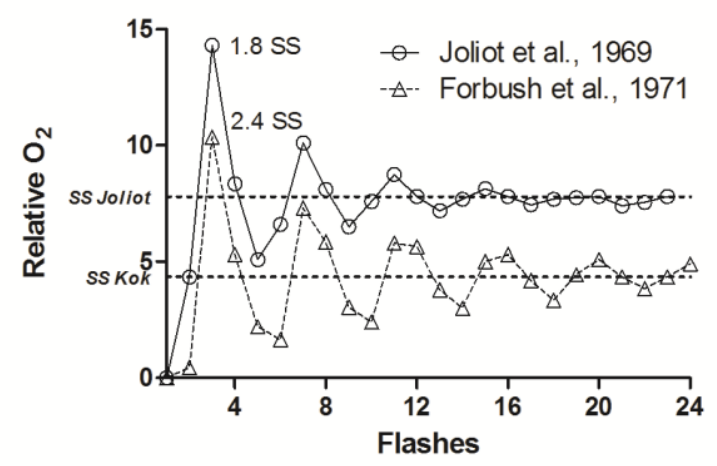

Idealized simulations

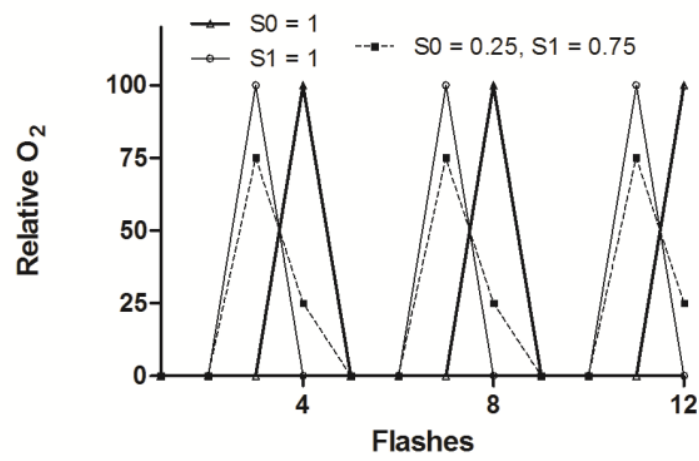

It can be seen from Figures SI1 through SI3 that several observations (and inferences thereof) noted by Kok were misplaced. There is no doubt that statistically, the third flash and its periodic repeats afforded high oxygen rates/yields in most cycles of "quartet flashes". (Figures SI2 \& SI3, panels a through i present the same data sets, the first for AUC analysis and the second for evolution in time.) However, in Figure SI1, Forbush's data (Kok's own group, on which the model was based!) shows that the peaks were centered on the fourth flash, fourth cycle onwards. In this regard, values within Figure SI2b/SI3b, SI2g/SI3g, SI2j etc. are other examples of reaction setups that negate the summated observations. In Figure SI2i, the $4^{\text {th }}$ flash gave the highest yield within the first cycle itself. The second flash within the first cycle gave significant values in several researchers" experiments (and this is not "very little or negligible" as Kok stated). In Figure SI1, one can clearly see that while a few peaks may coincide, the valleys don't. Also, the relative yields of values do not give a correspondence across experiments. For example- in Kok's data within Figure SIl, the $3^{\text {rd }} \& 4^{\text {th }}$ flashes (and their periodic repeats in the next two cycles) gave values higher than steady state rates whereas Joliot's data showed this trend only for the $3^{\text {rd }}$ flash (and its repeats in the next two cycles). Therefore, direct visualizations and deductions from Kok group's data and comparative analyses (with several researchers' data) do not support the prevailing "sequentially ordered and deterministic" paradigm.

Per the Kok model, the ratio of WSC population (S0:S1:S2:S3) in the initial state is in the ratio of 1:3:0:0 and the steady-state population is 1:1:1:1. So, in the first cycle, the fourth flash should have $\sim 1 / 3$ yield of the third flash (because first and second flashes gave little oxygen!). This requisite is flouted by practically every researcher's experiment, including Kok's own group's data. In Figure SI1 above for Kok's group's first cycle data, little oxygen evolution was seen in the first two flashes and the fourth flash gave $\geq 1 / 2$ the oxygen in comparison to the third flash. This must imply that very little double hits occurred in the first two flashes. If they did, we should have seen significant oxygen evolution in $2^{\text {nd }}$ flash itself. Therefore, in the third flash alone, the WSC experienced significant double hits. But then, if it did so, there would have been no way for any loss of oxygen here because a double hit would have taken the ETC through S4, which would have released the oxygen. Therefore, Kok's explanations (invoking a quartet of charged stable/labile states) do not account for the first cycle itself, in Kok's own datasets!

In Kok's data, the second and third cycle show that the $1^{\text {st }}$ flashes' (which corresponds to S3 state) yields are higher than the respective $2^{\text {nd }}$ flashes' (which corresponds to S2 state) yields. 
How could misses/double hits explain this outcome (given that going through transient S4 is obligatory!)? It can be seen that in several of these experimental systems, steady state is approached after $4^{\text {th }}$ cycle. Sometimes, even 6 cycles may not be enough to reach the steady state (as seen in Figure SI1). Also, if hits and misses were fairly randomized (which is what one would normally expect; given these should be clearly subject to the same parameters through several cycles), there should be little change in the S0 population, the S2 and S3 populations should only increase and S1 population should only decrease. It is very disconcerting to derive the "counter-theory" inference that the populations decay/evolve with rather unpredictable crossovers in several reactions (Figure SI3b, SI3f, SI3g).

In some experiments, the reason why the fourth quartet flashes go higher in oxygen yields (above the third quartet flashes, as the cycles go onwards to steady state) cannot be explained with the Kok model in a forthright way. (For doing so, one has to assume that the hits, misses and double hits have some rather skewed values for each one of these transition steps/experiments. That is what Kok's model solicits by assuming different $\alpha$ (index of misses) and $\beta$ (index of double hits) values for fitting each experimental data. Also, the initial stable values of the four states must be different in each setup [and not just S1(3):S0(1), as Kok assumed for a particular setup]. This practice would be similar to building and fitting a new theory to the experimental observations every time. We need to have a relatively simple theory that can explain a multitude of observations, at least on a qualitative scale. The much-acclaimed Kok's data fitting in Figure 1 of Forbush et al. (1971) has this problem evident (only the experimental values are shown in Figures SI1, SI2f and SI3f herein). While the experimental data shows peaks only at the $3^{\text {rd }}$ quartet flashes within the first three cycles, the fit model shows peaks at $3^{\text {rd }}, 3^{\text {rd }}$ and $4^{\text {th }}$ quartet flashes of cycles 1,2 and 3 respectively. Clearly, the observations display qualitative disagreement with the theoretical foundations. As seen, the ratio of steady state oxygen yield to the first cycle's $3^{\text {rd }}$ and $4^{\text {th }}$ flashes' yields, the number of cycles required for arriving at steady state, correspondence of relative rates within and across experiments, etc. varied significantly across groups and within groups. Given the deterministic theoretical basis of Kok's model (and ETC scheme), one would imagine that the above mentioned indices should have some common patterns and at least some experimentally observable "constant" signals should be derived. Clearly, the diverse observations do not conform to "expected reproducibility norms" that the currently prevailing deterministic ETC and oxygenesis scheme would dictate.

The only consistent observation that can be tangibly derived across different groups is- AUC for each cycle is practically constant (save the setups incorporating additives). This must mean that "misses" and "double hits" schemes are "quantitatively insignificant" in the overall scheme. While RC's 1-electron excitability is in small (sub-nano second) time-scales, its ability to get "double hit" depends on the "transferability" aspect, which is dependent on the slow 2-electron cycle of the bound/mobile quinones! [Also, double hits of S3 $\rightarrow \mathrm{S} 1$ are antithetic, not compatible with the ETC model. This is because it is not WSC that is directly under photo-electronic effects. It is the PSII's RC that is subjected to photonic excitations. In turn, the ETC that the RC is subjected to is inherently a one-electron transport system (without any transient pools to donate to or buffer with!) with two-electron transfers as rate-bottlenecks. An electron from WSC can move onward only if the ETC completes a full cycle. That is, a WSC in S3 state would have to go through S4 state before coming to S1 state. If it gets to S4 state, the connectivity breaks because oxygen is released and two more water molecules have to bond. Since the WSC is not 
subject to the photoelectric effect directly, double hits of this kind are merely "mathematical exercises" with little theoretical or experimental significance.]

Figure SI2: Cycle-wise analyses of oxygen evolution from pioneering researchers' data. In spite of the observations that the absolute value of amplitude changes, and also the peak within a quartet could lie on $3^{\text {rd }}$ or $4^{\text {th }}$ flash, the area under the curve $(A U C)$ remains constant through cycles (for experiments that did not incorporate additives), throughout the series of quartet flashes. AUC values are given for each cycle and the peak in each cycle is highlighted with a filled and emboldened symbol.
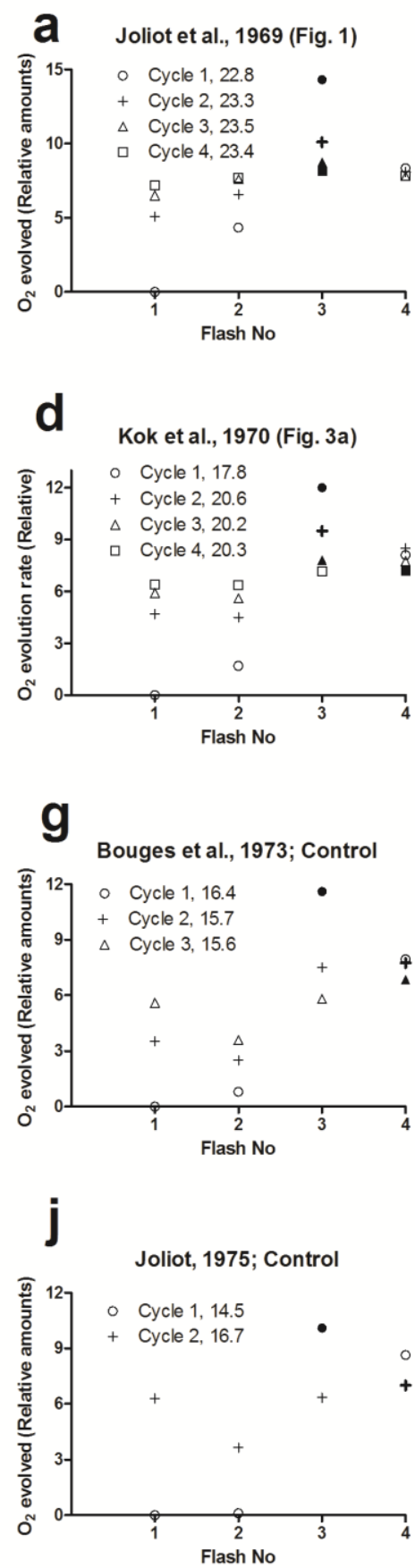
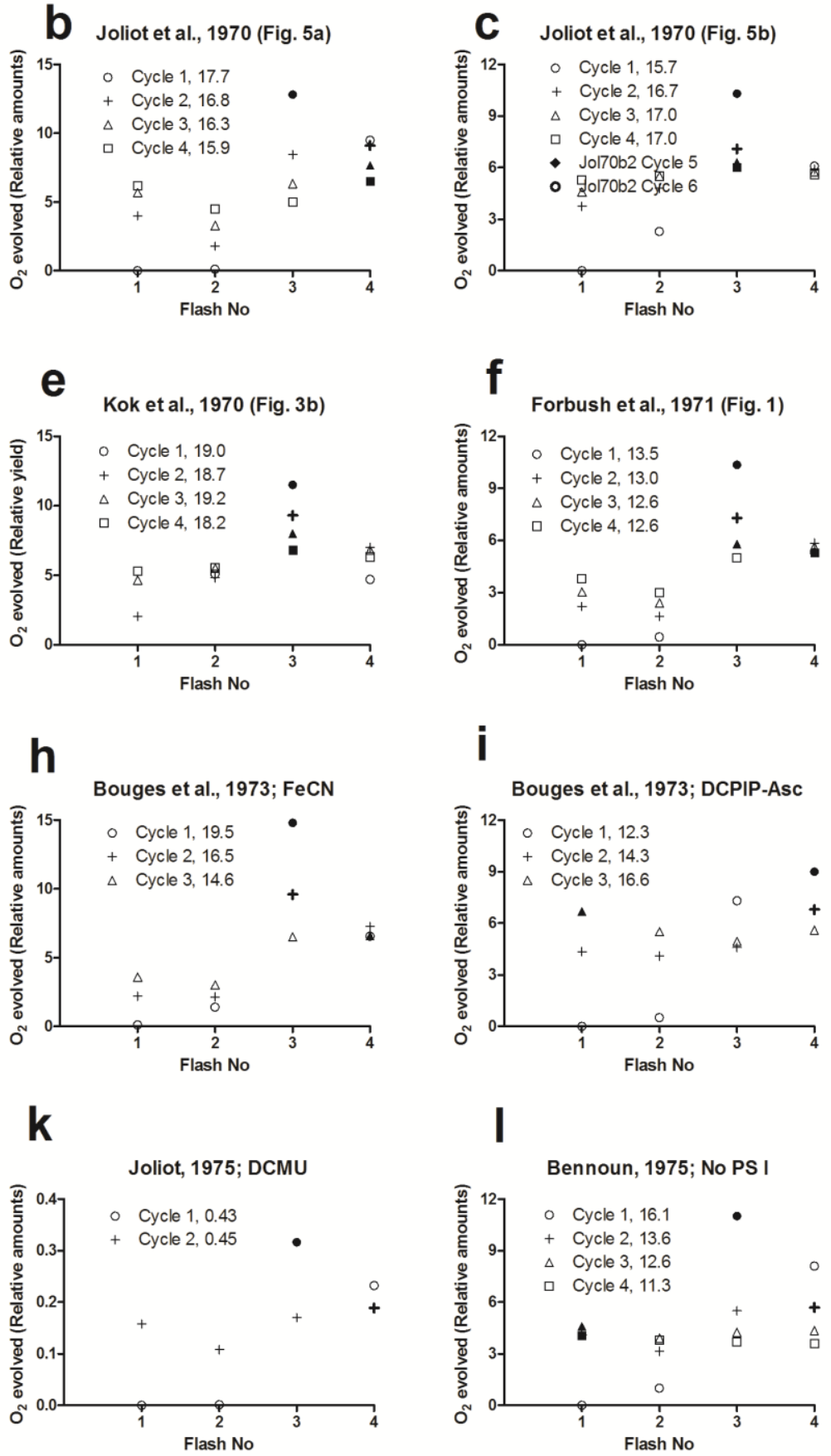
Figure SI3: Analyses of pioneers' data for temporal-phased oxygen evolution with respect to multiples of flash numbers 1 through 4. (The values of y axes in the panels of the first two rows are valid for comparison within the given panel only.)
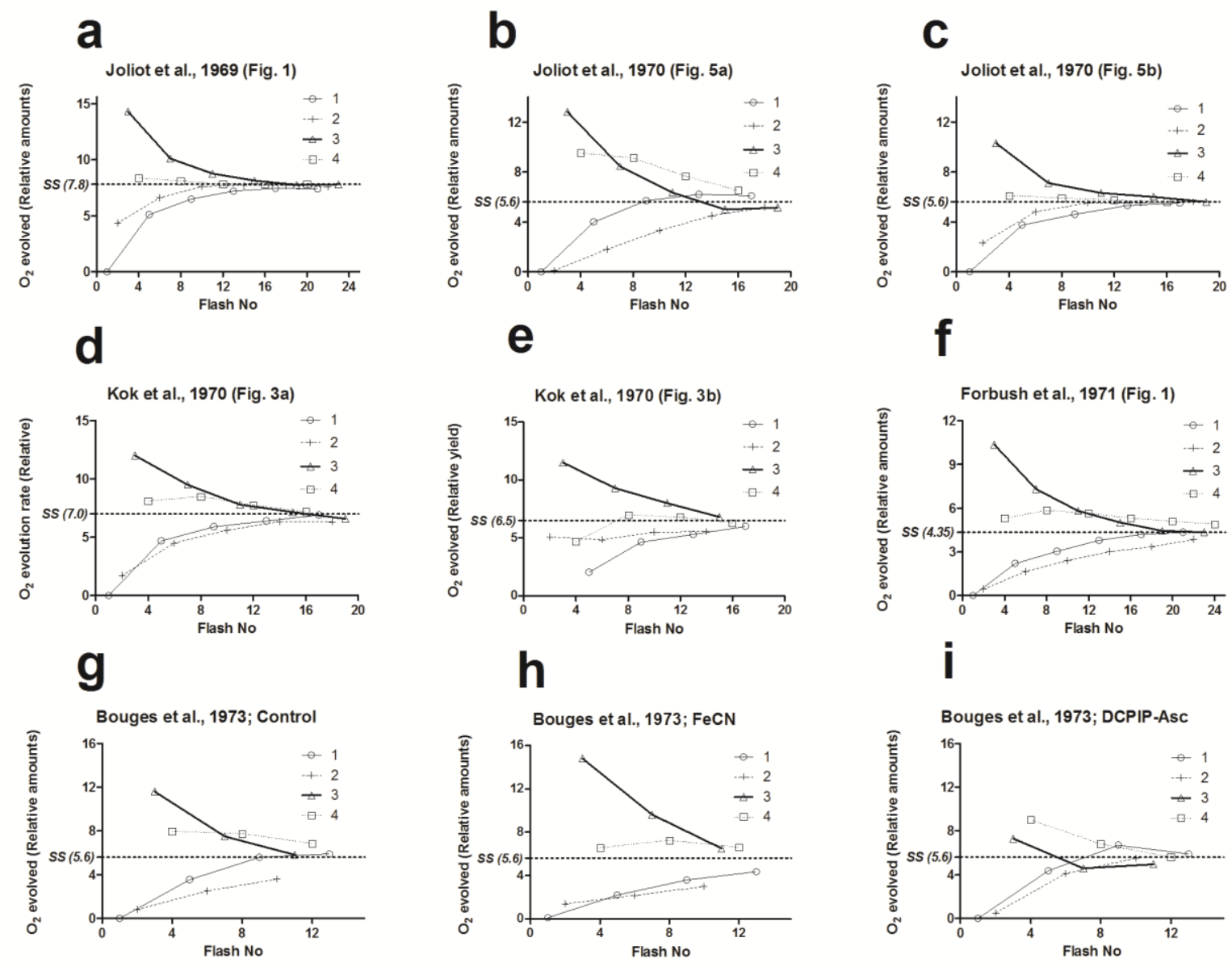

Very importantly, per the prevailing ETC/oxygenesis model, there is no scope for charge conservation in the system, because the relay agents are ordered and sequential (in series), with either single or double charge bearing capacities (excluding the WSC). (Also, the motility of quinones are limiting!) The current mechanistic purview does not permit charge to be retained or passed on from one cycle to another because each component is of a defined capacity and role. Saying that an initial photostimulation exercise enables the buildup of quinone pool is antithetical because there is nothing that stops the quinones from going through with the full cycle thereafter (which is supposed to take sub-millisecond time frames, compared to the seconds and minutes of the latency that ensues before the flashes are given!). Further, when the Kok-Joliot explanations were put up, the structures of the photosynthetic complexes were not known. The ability of the WSC/OEC to go through periodic cycles of electron transfer on the "hit/miss" of photons hitting RC of PS II is an untenable/unsubstantiated proposal. That is, the ETC connectivity through the Z scheme cannot be coordinated with the Kok-Joliot cycle, in any foreseeable way. This is because there is little physical connectivity for the hit/miss phenomenon 
to lead to a periodicity in the oxygen evolution profiles as seen in the Joliot cycles. Furthermore, this aspect is elaborated in the discussion below.

The dark adapted chloroplasts were primed with a suitable amount of light (a single flash followed by some "resting time"), before they were subjected to the series of flashes (numbered 1 onwards, as shown in Figure SI1) that were taken as "consequential". If a good starting point or consistency was desired with respect to experimental requisites, the only way it could have resulted is to have dark acclimatized chloroplasts to start off with, and having no "priming light" provision. If the explanation was based on a postulate that only one or two of the four WSC intermediates were initially stable, then what was the purpose of doing the pre-priming exercise, which would mess with that proposal of stability? Would not this "light priming" mess with the interpretations of periodicity and stability? This query is of paramount importance. The explanation afforded by Kok is that this single priming step affords the build-up of charged intermediates, so that a sequential relay can be set in thereafter. How can this be compatible with the theoretical assumptions of the ETC-oxygenesis cycles, per se? The assumption of prevailing ETC is founded on a linear charge transfer scheme with definite and sequential roles to each participant. The oxygenesis is supposed to be based on only four charge-able/discharge-able states of the system (and that being the WSC!). There are no generic roles or "buffering or reservoir" agents within. While the "intervening dark period" between flashes were in $\sim$ second scales, several intermediates' stability time-scales would be envisaged to be in sub-microsecond (or surely, in sub-millisecond scales!) time scales. Then, how is the assumption of " 4 distinct electronic states of WSC and their stabilities" used to explain the observation of arriving at a steady state from an initial periodicity? Most importantly, when the overall observation is supposed to be interpreted on a "one-flash, one electron" scheme, the explanation is based on "one-flash, zero/one/two electron" scheme (as the model assumes misses, single and double hits)! So, not only were the observations inconsistent with the explanations, the assumptions and explanations thereof were paradoxical and antithetical too! Thus, when scrutinized up close, it is seen that Joliot-Kok model is something quite like the chemiosmosis model- a mirage, neither making any holistic sense nor affording any purpose (other than fitting a curve to some equation).

Fig. 2 of Kok et al, 1970 is crucial to arrive at the crux of the issue here. It shows the oxygen evolution profiles by dark-acclimatized chloroplasts under a steady monochromatic light source, starting with nil or a single priming flash. The unprimed sample traced a sigmoid curve initially, significantly lagging behind the somewhat hyperbolic trace of the primed sample, although both traces achieved almost identical steady state values (>60 seconds). (Though the initial rate of the unprimed sample lagged behind the primed one, the former's trace caught up and exceeded the corresponding value of the primed sample, after crossing over at $\sim 5$ seconds.) The primed and non-primed samples reached their respective maximal values at $\leq 2$ and $\leq 8$ seconds. Within the first 10 seconds, both non-primed and primed samples showed higher oxygen evolution ( 7\% and $12 \%$ respectively) with respect to the steady state values. The protracted phase of the constant "steady state" value of oxygen evolution in the later times argues against inhibitions or deactivations. Therefore, it is clear that the correlation of oxygen detection to the "priming step", "maximal rate" and "steady state" is more complex than what is apparent. I have seen such timecourse profiles in simple heme-enzyme catalyzed peroxidation reactions. These profiles surely 
indicate a dynamic and temporally evolving interaction scheme of one-electron intermediates within milieu.

In Figure 1 of the very same paper by Kok et al (1970), the scenario analogous to the one shown in Figure 2 is extended to a series of priming flashes, from 0 to 7 (which have been duly labeled therein). It is seen that in almost all the samples, the steady state is achieved after $\sim 6$ seconds (save the unprimed sample, which achieves steady state after $\sim 8$ seconds). The point to note here is that samples that received 3 and 7 preceding flashes start off with higher oxygen evolution, which dips to lowered rates than the steady state values during the time period of $\sim 1$ to 5 seconds of illumination. Samples with 2 and 6 priming flashes show much higher values initially (than steady state yields), which falls rapidly as the illumination time increases, finally stabilizing at steady state values (never going lower than the steady state values). These data could now be compared to Figure SI3, where the oxygen evolution profiles of diverse workers have been presented as a function of the flash number (post the unique priming flash). The equivalent profiles are traces 1 (and 5), 2 (and 6), 3 (and 7), and 4 of Fig. 1 of Kok et al. (1970) and the connected profiles of traces numbered 1, 2, 3, and 4 of each one of the nine panels of the Figure SI2 of this manuscript. Even if we concede to Kok's inadmissible assumptions, the lack of one to one correspondence of data flouts the "stability of S0-S1-S2-S3 states of WSC" explanation.

An honest admission here, for the ensuing discussion: I am not confident about my concerns on protocols here because of a lack of "clear understanding" of the experimental procedures adopted. (The early papers by Joliot were in French and also, the experimental setups are unique to the field.) However, here are some samples of my doubts.

The rate calculations appear to be misplaced. How can initial rate be calculated with any experimental accuracy when the profiles are starting at different initial points? In the Figure 10 of Forbush et al, the data cannot be used to calculate rates. While curve 1 starts at a $\mathrm{Y}$ value of 0.2 , the curve 2 starts at 2.2. The value of $Y$ axis at the initial point of experimentation cannot be taken for rate calculation.

Joliot claims a response time of $10 \mathrm{~ms}$ for the electrode (Joliot \& Joliot, $1968 \mathrm{BBA}$ ) for the oxygen assay profile in Figures 7 and 8 of their paper. In Figure 7, Curve 1 is the unprimed sample, which is compared to Curves 2 and 3 in which light-priming was done with different setups. In all three experiments, the curves attain the amplitude of $\sim 5$ (Curve 1 takes $\sim 50 \mathrm{~ms}$ whereas curves 2 and 3 reach the amplitude in $\sim 15 \mathrm{~ms}$ and $\leq 10 \mathrm{~ms}$ respectively) and subsequently, all curves show identical decay profiles to reach the minimal value of $\sim 1$ after 1.8 seconds. Therefore, except for the time required to achieve the amplitude value, the time course of oxygen detection showed almost identical profiles for the two flashes, and only the "initial time" for attainment of the amplitude signal of the electrode changed across samples. I find that in the context, we needed to have an accurate measure of the yield of oxygen per flash, and not the initial rate per se. Else, we should have observed different amplitude values and decay curves for the different samples. Therefore, if the area under the electrode response decay curve is taken as the yield, it would give only a small variation of $<10 \%$ signal/output between the three experiments. At low temperature $\left(1^{\circ} \mathrm{C}\right),<400 \mathrm{~ms}$ was required for attaining the saturation value of oxygen released when subjecting chlorella cells to bright flashes of light [Emerson and Arnold (1932a)] and the reaction half life was $<40 \mathrm{~ms}$. At about $25{ }^{\circ} \mathrm{C}$, this half-life value can be 
approximated to be $\sim 4 \mathrm{~ms}$. Therefore, the overall reaction of oxygen evolution falls within the rough experimental range of the probe employed (whose response time was 10 millisecond). This would not be the best way to do an experiment! [And this would in turn mean that regardless of which flash number we consider, similar oxygen yield is obtained! Therefore, the multiplicity of "four illuminations leading to peak oxygen evolution" that was taken to be a yardstick for oxygen evolution, would be a sheer fallacy. Clearly, the first priming illumination(s) were taken out of the picture to derive an experimentally and theoretically aesthetic value (of quartets). Since oxygen evolution via the deterministic scheme would be from 2 molecules of water, a primed thought process had dictated the periodicity of oxygen evolution, and this expectation was fulfilled in the Kok-Joliot experiments/inferences.]

When we are talking about dark incubation times in $10^{3}$ seconds and flashes' separation times in seconds and electrodes response time in $10^{1}$ milliseconds and stabilities of the intermediates are the main consideration (which is currently believed to be less than millisecond ranges), my argument does not seem irrational. If the elementary 4-state model of Kok was true, we should have observations of prolonged oscillations, as defined by the idealized profiles in Figure SI1.

In the prevailing ETC scheme, there is no provision for conservation of electric charges from an initial state (whether dark acclimatized or primed with a definite amount of light) or across "quartet flash" cycles. There are no "electron traps" that are called upon to justify the priming flash. Therefore, the "conservation of charge" observation or explanation cannot be assumed or called upon for the prevailing ETC scheme. (One cannot invoke an antithetical assumption in the middle of a derivation!)

Joliot et al (1968) had computed a time period of $0.8 \mathrm{~ms}$ between the photostimulation event and release of oxygen. (Let us recapitulate that his process would necessitate a 4-electron relay through the ETC.) Kok et al (1969) and Stiehl and Witt (1969) reported a half-time of $\sim 10 \mathrm{~ms}$ for photoreduction of quinone pool. How could a reaction cycle with a slow bottleneck lead to a much faster reaction? Clearly, the purported elaborate Z-scheme ETC cannot be the operational mechanism!

Since the ETC cycles around in a time-frame of a few milliseconds, it can only process about a few hundred photons in a second. The same value would also be taken as the functional processing time within the RC. (Even if some excitation and charge transfers occur in nano-pico second time-scales, the electron transfers at several steps are in millisecond ranges. Since the system has finite resources, the steady state scenario would come to the afore-mentioned status. This would lead to the following statistical scenario: one chlorophyll molecule may only absorb a photon per second of the few hundred micromoles of photons that it would get.) Therefore, the prevailing assumptions cannot explain the observations. The conservation of charge can only be explained by a highly randomized/stochastic mechanistic model for oxygenesis, which does not have the sequential limitations of the current understanding.

Bouges et al (1973) data (Figure SI3g-i) shows the interesting observation that FeCN (ferricyanide, an oxidant) gave higher oxygen yields in the initial phases which went down as the cycles progressed, whereas the anti-oxidant ascorbate gave low AUC in the initial phase, which increased as cycles progressed. In the presence of ascorbate, the oxygen yield variation amongst 
flash numbers was lowered and the reaction approached steady state quicker, when compared to the control. The effect was quite the opposite in the presence of FeCN. This counters the specific electron transfer and relay concept of Z-scheme.

Per the Kok model, the state transitions theoretically occur within $10^{1}$ to $10^{3} \mu \mathrm{s}$ and the overall cycle itself gets completed within the same order of time as a/the limiting step $\left(10^{3} \mu \mathrm{s}\right)$. Experimentally, the flashes' durations are in the ranges of $10^{6} \mu \mathrm{s}$. Therefore, one could consider that there is ample scope for multiple hits and misses. However, in robust experimental systems and those lacking additives (Figures SI2 \& SI3, a through g, and j), the area under the curve (AUC) analysis for each cycle remained constant ( $\pm 15 \%$ variation, within a given experiment). This statement negates the assumptions/interpretations for the introduction of constants like $\alpha$ and $\beta$ (accounting for the so-called misses and double hits) for explaining the periodic waning and "lowering amplitude". That is- there were no losses within the system as it approached the steady state (and "damping of amplitudes" would only be expected in a system with several states and finite probabilities for crossovers). If there is any kind of miss (with respect to any significant/expected event), we cannot note a conservation of charge through the cycles. The system is in dynamic equilibrium from the starting state until it reaches the steady state (and beyond). Further, though multiple hits seem a theoretical possibility with respect to the excitability of a photosystem, relay of an electron from water to the reaction center must occur within microsecond time scales.

Before we undertake the exercise to explain the Joliot-Kok experiments' findings, it is imperative that one understands that a given set of experimental observations could be fit into a wide variety of mathematical equations/theories (with appreciable $\mathrm{R}^{2}$ values) and such a fitting of experimental data is not a projection/prediction of accuracy of the hypothesis/data. For example (as shown in Figure SI4), when a simple non-linear regression analysis of concentration versus activity is taken, one can simulate a Michaelis-Menten equation for experimental data (of 10 points) varying through typically one decade of concentration. Now, this is the equation of a typical hyperbola, which can be easily fit into a parabolic (shown here) or elliptical/catenary curves (not shown). Unlike the assumptions involved in M-M kinetics, totally irrelevant exponential associations with one or two binding site considerations could also give excellent fits to the data points. So, how does one know which of these fits could be correct? Only by a meticulous and critical analyses of both the theoretical foundations and experimental data, we can approach a more "truthful" and reasonable explanation.

Therefore, what Kok achieved is the fitting an experimental data curve to a quantitative expression/model based on certain mechanistic assumptions that have little grounding in reality. Vladimir Shinkarev's (Biophys. J. 2003) Markov chain-based solution for the graphical problem has a stochastic/probabilistic approach. This is definitely an appealing mathematical strategy that could explain the rather "chaotic" results. However, for having a solid theoretical relevance, we must resort to a different set of starting assumptions and systemic parameters/variables. In which case, it would then be mandated that the outcome therein is interpreted in a different way too.

Figure SI4: The points correlating and connecting a theory with experiment, a graphical argument 

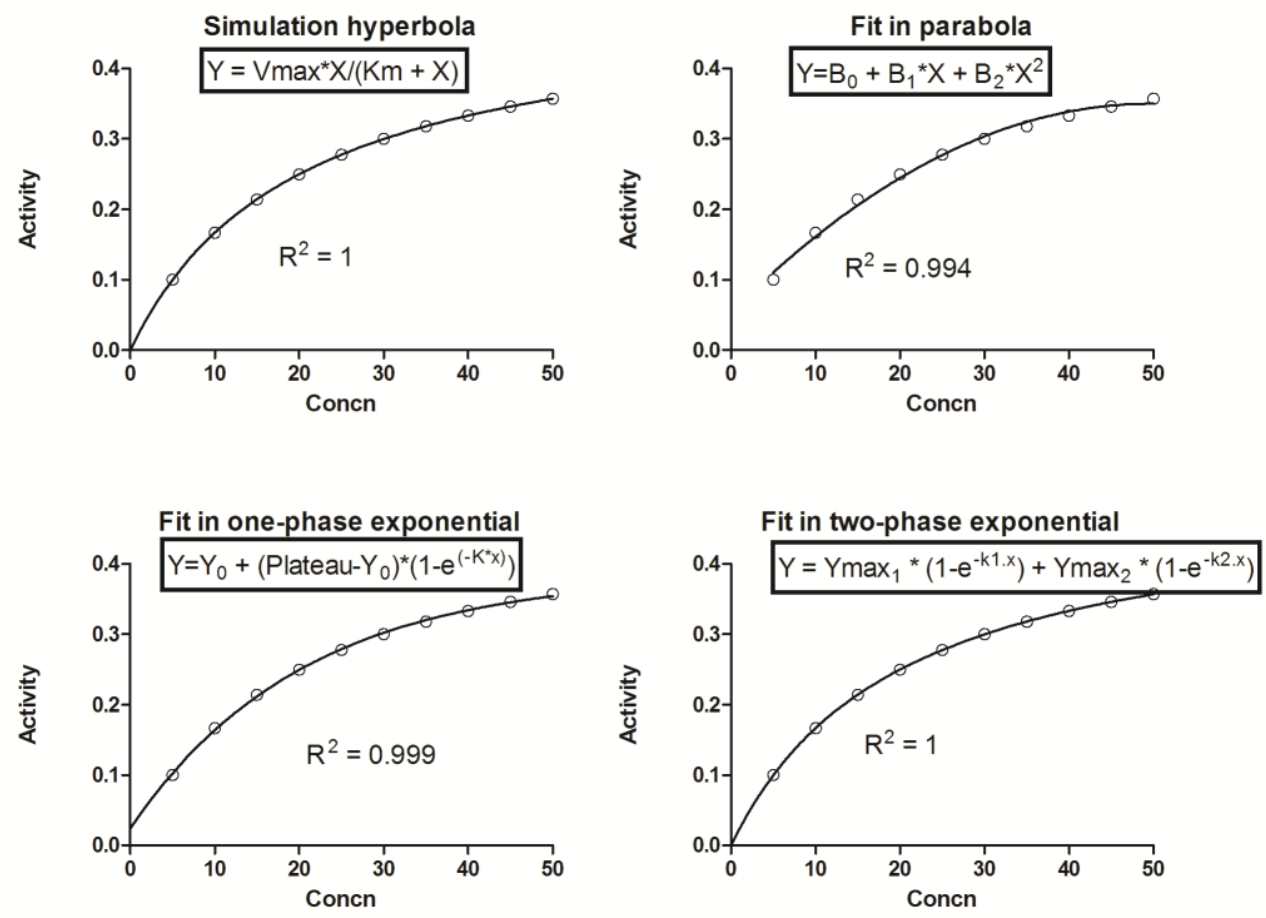

Figure SI5 below shows simple evidence to the statements above. In a reaction system with faulty or dysfunctional PS I, there was no steady-state achievement, but a gradual decrease in oxygen evolution rate over cycles. Clearly, per the prevailing concepts, oxygen evolution is a function of PS II alone and we see that the periodicity is no more, even at the second cycle (even if we overlook the production of oxygen in the $2^{\text {nd }}$ flash of the first cycle)! Such observations dictate the application of alternate theoretical assumptions.

Figure SI5: Oxygen evolution in a modified Z-scheme setup

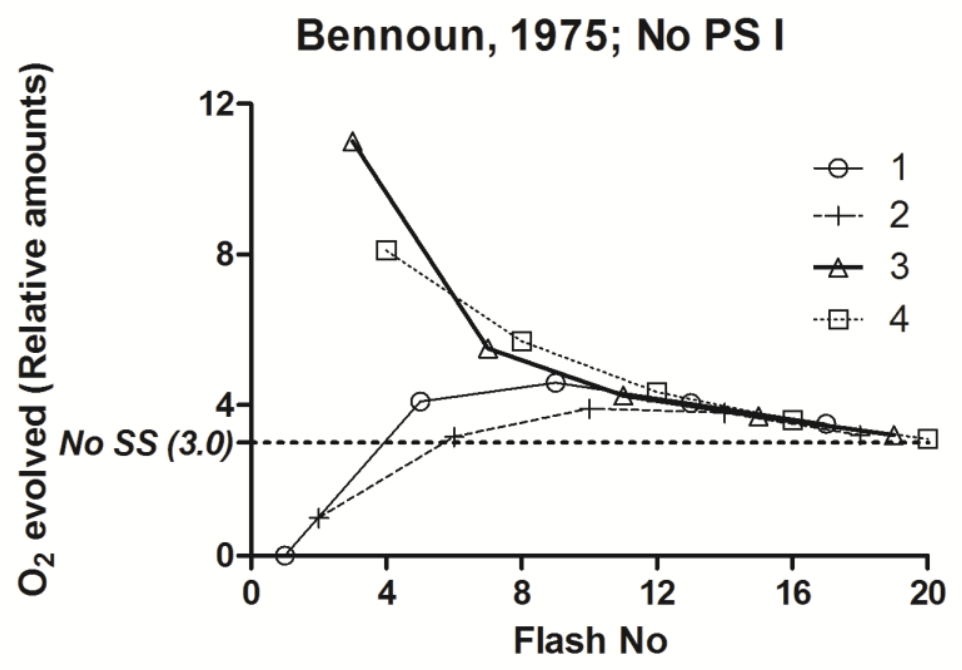

